\title{
Effect of Intra-Row Spacing and Cropping System with Sugar Beet on Growth, Yield and Quality of Two Garlic Cultivars
}

\author{
Naglaa H. Hussien ${ }^{1}$, Moshira, A. EL-Shamy²
}

\begin{abstract}
A field experiment was carried out at Sakha Horticulture Research Station, Kafr El- Sheikh Governorate Egypt, during winter seasons of 2014/2015 and 2015/2016 to study the effect of two garlic cultivars, two intra-row spacing and three intercropping systems addition sole were used on growth, yield and quality of garlic and sugar beet, as well as to evaluate land equivalent ratio (LER) and economic return under different combinations. Variety Geloria sugar beet (a mono-germ variety) was used as the main crop. The experiment was laid out in a split-split plot arrangement in a randomized complete block design. Main plots contained garlic cultivars (Balady cv. and Sids-40 cv.), sub plots were devoted to intra-row spacing $(10 \mathrm{~cm}$ and $15 \mathrm{~cm})$ and subsub plots were assigned to intercropping system (one garlic row, two garlic rows and three garlic rows), pure stand of sugar beet and also pure stand of garlic. Results indicated that Balady cv. was surpasses Sids-40 of germination \%, plant height, bulb diameter and clove number. Moreover, Sids-40 cv. gave the highest values for all the other studied characteristics (leaves number, fresh and cured bulb weight, clove weight and total yield per fed.) in two seasons. The $15 \mathrm{~cm}$ intra-row spacing superior over the 10 cm intra-row spacing in most characteristics. Also, one garlic row resulted the highest improvement of all characteristics except total yield per feddan of garlic and sugar beet. However, the pure stand of garlic and sugar beet had the highest values of total yield fed $^{-1}$ and its components in comparison to intercropping system. The highest LER were obtained from Balady cv. at $10 \mathrm{~cm}$ intrarow spacing with three garlic rows, followed by the same cultivar at $15 \mathrm{~cm}$ intra- row spacing with two rows $(1.55$ and 1.51), respectively, as mean of both seasons. The highest net income was obtained from Sids-40 cv. (20052 L.E.) followed by Balady cv.(19355L.E) at $15 \mathrm{~cm}$ intra row spacing with two rows as mean of both two seasons.
\end{abstract}

Keywords: Garlic, Allium Sativum 1., Cultivar, IntraRow Spacing, Intercropping, Sugar Beet, Productivity, Quality

\section{INTRODUCTION}

Garlic (Allium Sativum L.) is one of the most important vegetable crops in the world (Baghalian, 2005). It's belonging to family Alliaceae and Genus Allium (Dayi, 2008).As well as, have Anti-infective properties such as anti-bacterial, antifungal, anti-cancer, lowering blood sugar and blood lipids, power suppliers, also reduce blood platelet aggregation. In terms of production, garlic is coming second after onion, it is a bulbous plant, and produce a bulb which consists of bulblets called cloves (valadez, 1992). Garlic is a high value cash crop due to its various used in local consumption, food processing and exportation (ALOtayk et al., 2008 \& EL eshmwiy et al., 2010). In Egypt, the cultivated area is about 20532 feddan with average yield of 9.7 ton/fed. in 2014 year (Bulletin of the Agriculture Statistics, Economic Affairs Sector. Part 1 winter crops. February 2013/2014 Ministry of Agriculture and Land Reclamation)

Garlic bulbs yield and quality are changed with cultural practices, climate and used varieties (Abdelrazzak \& El sharkawy 2013). Nasser et al. (1972), stated that the Chinese cultivar proved to be of high yielding ability, more uniform cloves of heavier weight and fewer cloves number per bulb than local variety (Balady cv.). Moustafa et al. (2009), evaluated some new imported cultivar under the Egyptian conditions and showed that there were significant differences among the tested genotypes. Mohamed (2004) \& Ammar (2007) found that, Balady cultivar significantly superior Sids-40 cultivar in plant height but Sids-40 cultivar produced more leaves. Hussein et al., (1995), reflect to Chinese cultivars gave better bulb quality (bulb diameter, bulb weight). Significant variations were observed for various garlic characteristics as reported by Osman \& Moustafa (2009) \& Aly, Shreen (2010). Sandhu et al. (2015) reported that the analysis of variance showed significant difference among all the genotypes for all the characters under study.

Plant spacing influences the growth and yield of garlic. Yield of garlic is dependent on the number of plants accommodated per unit area of land (Alam et al., 2010). Several authors reported that, increasing the yield and improved the grade of garlic bulb due to proper plant spacing, wider spacing increased greater plant height and number of leaves (Purewal \& Daragan, 1961; Om and Srivastava, 1977), increased the bulb size (Menezes et al. 1974) and reduced the yield of garlic (Rahim et al., 1984). The increased number of plant per unit area in closer spacing compensates the loss of reduced bulb sizes and ultimately increases the yield. Castellanos et al., (2004), reported that, although plant yield increased when the higher plant density were used

${ }^{1}$ Potato \& Vegetatively Propagation Vegetables Dept.

Hort. Res. Inst., Agric. Res. Cen., Giza, Egypt.

${ }^{2}$ Crop intensification Res. Dep., Field Crops Res. Institute, ARC, Egypt

Received June 6, 2017, Accepted June29, 2017 
but the yield improvement increased plant stand is offset by the reduction in bulb size which severely affects quality and market value, when garlic is produced for fresh market. Castillo et al. (1996), recommended handling plant densities from 140000 to 180000 plants/ha to ensure a good bulb diameter. Olfati. et al., (2016), showed that, moderate between row spacing and plant density for fresh market garlic and low between row spacing and high plant density for higher yield is proposed.

Intercropping is old practice of growing two or more crops simultaneously in the same piece of land. It is a technique of crop intensification in both space and time, the competition between crops may occur during a part or whole of crop growth period. The relationships of cooperation and competition are density dependent (Donald, 1963). At low densities, there is cooperation but, the active competition comes into existence at high densities. The competition between base and intercrops essentially depends on the maturity period, rooting habit, pattern of canopy spread, etc. It is proven fact that the utilization of natural resources viz. space, light, soil moisture, air, nutrient etc. were more efficient with intercropping than sole cropping ( Lomte \& Dabhade, 1990). Sarkar et al. (1995), showed that intercropping not only stabilizes crop production by reducing the impact of weather vagaries, but also increases cropping intensity considerably. In farming systems with low external inputs, intercropping became also appeared as an economically viable option for an integrated weed management (Teasdale, 1998, Schoofs \& Entz, 2000 and Jabbar et al. 2010). The benefit of using garlic in mixed cropping is that it effectively repels harmful pests while retaining beneficial ones (Amin et al., 2011).

Sugar beet (Beta vulgaris L.), is the second source of sucrose all over the world and in Egypt. As well as, Sohier and Ouda (2001), revealed that the importance of sugar beet crop to agriculture is not confined only to sugar production, but also it is adapted to saline, calcareous and sodic soil. Moreover, as a short duration and quires less water to produce kilogram of sugar requires about $1.4 \mathrm{~m}^{3}$ of water. It's a deep-rooting crop (up to $3 \mathrm{~m}$ ) so the crops like Onion and garlic are very much suite to intercropping with sugar beet. Since these crops are shallow rooted bulbs and having low canopy, so they do not compete with space and deep-rooted long duration crop (El-Sarag, 2009; Mahmoud et al., 2012 \& Masri et al., 2015). Toaima et al., (2000), reflect to the Intercropping patterns of sugar beet + garlic or sugar beet + onion improves the growth traits, yield and other components of sugar beet. The area of sugar beet production had increased in the recent years (16900 fed. in 1982 season to 450000 fed. in 2012 season), and the contribution of sugar beet to sugar production increased largely, as it reached $35.5 \%$ of the total sugar production in 2012 season (FAO, 2012).

In Egypt, the agricultural system has one of main problems. It's the low area of cultivated land per grower about $44 \%$ of the growers own or works in an area of one feddan or less (Ahmed et al., 2009). In view of lessening resources like water, energy and arable of land for feed, there are a dire requirement new strategies and techniques of crop production to meet the needs of expanding for food, feed and fiber.

The objective of this study was to evaluate the impact of two garlic cultivars and two plant spacing under garlic intercropping system with sugar beet, as well as land equivalent ratio (LER) and monetary returns during garlic and sugar beet production and total income, under Egyptian conditions.

\section{MATERIALS AND METHODS}

A field experiment was carried out at Sakha Horticulture Research Station farm, Kafr El- Sheikh Governorate Egypt, during winter seasons of 2014/2015 and 2015/2016. Experiment location was in the middle Northern Part of Nile Delta region in along the western branch of the Nile. The site elevation of about 6 meters above mean sea level and it lies at $30.57 \mathrm{~N}$. Latitude, 31.07 E. longitudes. The experiment soil texture was clay loam in both seasons (Table a).

A split-split plot in a randomized complete block design arrangement with three replications was used. Garlic cultivars were allocated to main plots, meanwhile, the sub plots were assigned for two plant spacing as three intercropping systems with sole garlic and sugar beet were distributed at sub-sub plots. As follow:-

Main plots: garlic cultivars were Balady cv. and Sids$40 \mathrm{cv}$.

Sub plots : intra-row spacing were 10 and $15 \mathrm{~cm}$.

Sub-sub plots: intercropping system were as follow:-

1 - Sole garlic as monoculture.

2- Sole sugar beet as monoculture.

3- One garlic row was planted on the top of sugar beet bed.

4- Two garlic rows were planted on the top of sugar beet bed.

5- Three garlic rows were planted on the top of sugar beet bed. 
Table a. Some properties of the experimental soil

\begin{tabular}{clllll}
\hline Texture & Clay \% & Silt \% & Total sand \% & EC dS.m & PH \\
\hline Clay loam & 59.01 & 26.2 & 14.8 & 2.1 & 8.1 \\
\hline
\end{tabular}

Each experimental plot includes five ridges $4 \mathrm{~m}$ length and $1.20 \mathrm{~m}$ width with an area $24 \mathrm{~m}^{2}$. Sugar beet variety Geloria (a mono-germ variety) was used as main crop. Seeds of sugar beet (received from Sugar Crops Res. Inst.) were planted on $7^{\text {th }}$ and $8^{\text {th }}$ October for the $1^{\text {st }}$ and $2^{\text {nd }}$ seasons respectively. Garlic bulbs for each cultivar (obtained from Hort. Res. Institute, Agri. Res. Center, Giza), were carefully separated into individual cloves. Cloves were soaked overnight in tap water before planting. The principal target of water to enhance sprouting, then the cloves were sown on October $8^{\text {th }}$ and $9^{\text {th }}$ for the first and second seasons and they harvested on $28^{\text {th }}$ April and $10^{\text {th }}$ May for two seasons when older leaves turned yellowish green and had started withering. The land of the experimental field was prepared as recommended, and all the agricultural practices for sugar beet were applied according to the recommendations of the Egyptian Ministry of Agriculture.

\section{Data record}

a- Growth, yield and its components parameter of garlic plants:

1-Germination percentage of garlic plants: Germination percentage of garlic plants after 30 to 40 days from planting date were recorded. The percentage of germinated cloves were counted by using the following equation:

$$
\text { Germination } \%=\frac{\text { Number } \quad \text { of sprouted }}{\text { cloves } / \text { plot }} \times 100
$$

2. Plant height $(\mathbf{c m})$ : were taken randomly from average of ten plants/plots.

3. Leaves number $\operatorname{plant}^{-1}$ : number of leaves calculated as the average number of green leaves.

4- Leaf area $\left(\mathbf{c m}^{2}\right)$ : The method calculates the leaf area as a product of leaf length, leaf width and a correction factor (0.72) according to the leaf parameter method by Džamić et al. (2001) and Djordje M. et al. (2011).

5. Bulb diameter plant ${ }^{-1}$ : Average bulb diameters per plant in each treatment were measured in centimeters.

6. Fresh and curd bulb weight plant ${ }^{-1}$ : Average bulb weight per plant in each treatment was calculated in grams by the use of the following equation:

Average bulb weight $=\frac{\text { Total weigth of bulbs }}{\text { Total number of bulbs }}$
7- Number of cloves bulb ${ }^{-1}$.

8- Average of clove weight.

9- Total yield (ton/fed.): After harvesting, the yield of each plot was left in the farm as intact plant (with tops and roots) for fifteen days until the curing process was completed. After wards the yield was weighted and expressed as ton/feddan.

B- Sugar beet growth, yield and its components parameters: It included leaves number/plant, leaf fresh weight/ plant $(\mathrm{g})$, root fresh weight $(\mathrm{g})$, root length $(\mathrm{cm})$, root diameter $(\mathrm{cm})$, root weight/ plant $(\mathrm{g})$, and total root yield (ton/fed).

c- Sugar beet quality parameters: They included Sugar and purity (sucrose $\%$ and purity $\%$ ) content in roots were determined using an automatic sugar polarimeter according to Le-Docte (1971) method as described by Mc Ginnus (1982).

Economic feasibility: Total yield of sugar beet of intercrops and garlic of intercrops, as compared with sole crop were recorded at harvest and cost benefit ratio worked out. Gross return (L.E. fed ): Gross return from each treatment was calculated in Egyptian pounds (L.E.).

Price of ton of sugar beet was obtained by Egyptian Sugar and Integrated Industries Company and price of ton of garlic was obtained by market search.

Land Equivalent Ratio: According to Willy and Osiru (1972), land Equivalent Ratio (LER) was determined as follows:

$$
\mathrm{LER}=\frac{Y a b}{Y a a}+\frac{Y b a}{Y b b}
$$

Where:

$Y_{a a}$ and $Y_{b b}$ are the sole yield of $a$ and $b$ crops, respectively, ton/fed.

$\mathrm{Y}_{\mathrm{ab}}$ is yield of the intercropped a crop, ton/fed.

$\mathrm{Y}_{\mathrm{ba}}$ is yield of the intercropped $\mathrm{b}$ crop, ton/fed.

\section{Statistical analysis:}

The obtained data were submitted to analysis of variance according to the method described by Gomez and Gomez (1984). Treatment means were compared by Duncan's Multiple Range Test (Duncan, 1955). 


\section{RERSULTS AND DISCUSSION}

*Performance of garlic:-

\section{Main effects:}

1- Effect of garlic cultivars:

1-a- Germination percentage and some vegetative growth parameters:

Results in Table (1) showed the effect of garlic cultivars, plant spacing and cropping system on germination percentage and vegetative growth components of garlic in winter seasons of 2014/2015 and $2015 / 2016$. The results revealed that garlic cultivars exhibited significant effect on germination percentage, leaves number and plant height traits. Balady cv. gave the highest values of germination \% (82.28 and 80.67) and plant height (74.06 and 72.6) in two seasons respectively. On contrary, Sids-40 cv. gave the highest value of leaves number trait (9.67 and 8.78) in the two seasons respectively.

The obtained results are in harmony with those obtained by Moustafa et al, (2009) and Aly (2010) who indicated that the variation of different garlic cultivars in their emergence \%. Also, agree with Al-Otayk et al. (2008), Anwar and Gouda (2012) and Abdel Razzak and El-Sharkawy (2013), Panse (2013), El Nagar and ElZohiri (2015) and Azza and Naglaa (2016). On the other hand, two garlic cultivars recorded insignificant differences on leaf area trait in both seasons.

\section{1-b- Yield and its components:}

Data presented in table (2) showed the effect of garlic cultivars, plant spacing and cropping system on garlic yield and quality in seasons 2014/2015 and 2015/2016. Cultivars significantly differed in bulb diameter per plant $(\mathrm{cm})$, fresh and cured bulb weight $(\mathrm{g})$, cloves number per bulb, clove weight $(\mathrm{g})$ and total yield (ton/fed.). The results indicated that, the highest values in bulb diameter per plant (5.64 and $5.59 \mathrm{~cm} / \mathrm{bulb})$ and cloves number per bulb (50.49 and 51.00) in two seasons respectively were recorded from Balady cultivar. On the other hand, Sids-40 cv. significantly surpassed Balady cv. in fresh bulb weight (66.61 and 64.67), cured bulb weight (30.28and $28.11 \mathrm{~g} / \mathrm{bulb}$ ), clove weight (3.34 and $3.31 \mathrm{~g} /$ clove) and total yield (4.94 and 4.79 ton/fed.) in two seasons respectively.

These results agree with those obtained by Hussein, et al., (1995), Al-Otayk et al., (2008), abou el-magd et al., (2012) and Anwar and Gouda (2012) they found that the lowest bulb, cloves weight and total yield were obtained in Balady cultivar.

\section{2- Effect of garlic plant spacing:}

Plant density changed when garlic cultured with different intra-row spacing. Previous studies carried out on plant density indicate direct influence on growth, yield and its components as Castellanos et al.,(2004).

Table1. Effect of garlic cultivars, plant spacing and cropping system on garlic germination percentage and some vegetative growth components

\begin{tabular}{|c|c|c|c|c|c|}
\hline Treatments & & Germination \% & $\begin{array}{l}\text { leaves number } \\
\text { plant }^{-1}\end{array}$ & $\begin{array}{l}\text { Plant } \\
\text { height }(\mathrm{cm})\end{array}$ & $\begin{array}{r}\text { Leaf area } \\
\left(\mathrm{cm}^{2}\right)\end{array}$ \\
\hline \multicolumn{6}{|c|}{$2014 / 2015$} \\
\hline \multirow{2}{*}{ Cultivars } & Balady & $82.28^{\mathrm{a}}$ & $8.96^{\mathrm{b}}$ & $74.06^{\mathrm{a}}$ & $75.05^{\mathrm{a}}$ \\
\hline & Sids-40 & $80.64^{b}$ & $9.67^{\mathrm{a}}$ & $66.49^{\mathrm{b}}$ & $79.62^{\mathrm{a}}$ \\
\hline \multirow{2}{*}{ Distances } & $10 \mathrm{~cm}$ & $81.17^{\mathrm{b}}$ & $9.15^{\mathrm{b}}$ & $68.44^{\mathrm{b}}$ & $73.53^{b}$ \\
\hline & $15 \mathrm{~cm}$ & $81.75^{\mathrm{a}}$ & $9.48^{\mathrm{a}}$ & $72.11^{\mathrm{a}}$ & $81.14^{\mathrm{a}}$ \\
\hline \multirow{4}{*}{ Cropping system } & Sole & $82.16^{\mathrm{a}}$ & $10.6^{\mathrm{a}}$ & $79.26^{\mathrm{a}}$ & $96.44^{\mathrm{a}}$ \\
\hline & One row & $81.69^{\mathrm{ab}}$ & $9.85^{\mathrm{b}}$ & $73.93^{b}$ & $85.11^{\mathrm{b}}$ \\
\hline & Two rows & $81.42^{b}$ & $8.82^{\mathrm{c}}$ & $66.98^{\mathrm{c}}$ & $69.77^{\mathrm{c}}$ \\
\hline & Three rows & $80.58^{\mathrm{c}}$ & $8.01^{\mathrm{d}}$ & $60.93^{\mathrm{d}}$ & $58.02^{\mathrm{d}}$ \\
\hline \multicolumn{6}{|c|}{$2015 / 2016$} \\
\hline \multirow{2}{*}{ Cultivars } & Balady & $80.67^{\mathrm{a}}$ & $8.15^{\mathrm{b}}$ & $72.60^{\mathrm{a}}$ & $62.03^{\mathrm{a}}$ \\
\hline & Sids-40 & $79.06^{\mathrm{b}}$ & $8.78^{\mathrm{a}}$ & $65.19^{\mathrm{b}}$ & $65.79^{a}$ \\
\hline \multirow{2}{*}{ Distances } & $10 \mathrm{~cm}$ & $79.58^{\mathrm{b}}$ & $8.32^{\mathrm{b}}$ & $67.10^{\mathrm{b}}$ & $60.77^{\mathrm{b}}$ \\
\hline & $15 \mathrm{~cm}$ & $80.15^{\mathrm{a}}$ & $8.62^{\mathrm{a}}$ & $70.69^{\mathrm{a}}$ & $67.06^{\mathrm{a}}$ \\
\hline \multirow{4}{*}{ Cropping system } & Sole & $80.54^{\mathrm{a}}$ & $9.62^{\mathrm{a}}$ & $77.71^{\mathrm{a}}$ & $79.70^{\mathrm{a}}$ \\
\hline & One row & $80.09^{\mathrm{ab}}$ & $8.96^{\mathrm{b}}$ & $72.48^{\mathrm{b}}$ & $70.34^{\mathrm{b}}$ \\
\hline & Two rows & $79.83^{b}$ & $8.02^{c}$ & $65.67^{\mathrm{c}}$ & $57.66^{\mathrm{c}}$ \\
\hline & Three rows & $78.99^{c}$ & $7.28^{\mathrm{d}}$ & $59.73^{d}$ & $47.95^{\mathrm{d}}$ \\
\hline
\end{tabular}

Means followed by the same letters were not significantly differed according to Duncan's multiple range test. 
Table 2. Effect of garlic cultivars, plant spacing and cropping system on garlic yield and quality

\begin{tabular}{|c|c|c|c|c|c|c|c|}
\hline \multicolumn{2}{|c|}{ Treatments } & \begin{tabular}{l}
\multicolumn{1}{c}{ Bulb } \\
Diameter \\
$(\mathbf{c m})$
\end{tabular} & $\begin{array}{c}\text { Fresh bulb } \\
\text { Weight } \\
\text { (g) }\end{array}$ & $\begin{array}{c}\text { Cured bulb } \\
\text { Weight } \\
\text { (g) }\end{array}$ & 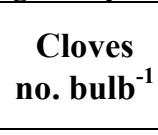 & $\begin{array}{c}\text { Clove } \\
\text { weight } \\
\text { (g) }\end{array}$ & $\begin{array}{l}\text { Yield } \\
\text { ton/fed }\end{array}$ \\
\hline \multicolumn{8}{|c|}{$2014 / 2015$} \\
\hline \multirow{2}{*}{ Cultivars } & Balady & $5.64^{\mathrm{a}}$ & $60.02^{\mathrm{b}}$ & $27.28^{\mathrm{b}}$ & $50.49^{\mathrm{a}}$ & $1.30^{\mathrm{b}}$ & $4.43^{\mathrm{b}}$ \\
\hline & Sids-40 & $5.11^{\mathrm{b}}$ & $66.61^{\mathrm{a}}$ & $30.28^{\mathrm{a}}$ & $14.31^{\mathrm{b}}$ & $3.34^{\mathrm{a}}$ & $4.94^{\mathrm{a}}$ \\
\hline \multirow{2}{*}{ Distances } & $10 \mathrm{~cm}$ & $5.24^{b}$ & $61.26^{\mathrm{b}}$ & $27.85^{\mathrm{b}}$ & $32.41 \mathrm{a}$ & $2.26^{\mathrm{b}}$ & $4.96^{\mathrm{a}}$ \\
\hline & $15 \mathrm{~cm}$ & $5.50^{\mathrm{a}}$ & $65.35^{\mathrm{a}}$ & $29.7^{\mathrm{a}}$ & $32.40 \mathrm{a}$ & $2.38^{\mathrm{a}}$ & $4.40^{\mathrm{b}}$ \\
\hline \multirow{4}{*}{$\begin{array}{l}\text { Cropping } \\
\text { system }\end{array}$} & Sole & $6.01^{\mathrm{a}}$ & $72.12^{\mathrm{a}}$ & $32.76^{\mathrm{a}}$ & $32.95 \mathrm{a}$ & $2.79^{\mathrm{a}}$ & $6.52^{\mathrm{a}}$ \\
\hline & One row & $5.63^{\mathrm{b}}$ & $66.9^{\mathrm{b}}$ & $30.4^{\mathrm{b}}$ & $32.70 \mathrm{a}$ & $2.62^{\mathrm{b}}$ & $2.34^{\mathrm{d}}$ \\
\hline & Two rows & $5.14^{\mathrm{c}}$ & $59.6^{\mathrm{c}}$ & $27.08^{\mathrm{c}}$ & $32.44 \mathrm{a}$ & $2.31^{\mathrm{c}}$ & $4.22^{c}$ \\
\hline & Three rows & $4.71^{\mathrm{d}}$ & $54.7^{\mathrm{d}}$ & $24.88^{\mathrm{d}}$ & $31.52 \mathrm{a}$ & $1.56^{\mathrm{d}}$ & $5.64^{\mathrm{b}}$ \\
\hline \multicolumn{8}{|c|}{$2015 / 2016$} \\
\hline \multirow{2}{*}{ Cultivars } & Balady & $5.59^{\mathrm{a}}$ & $58.27^{\mathrm{b}}$ & $25.33^{b}$ & $51.00^{\mathrm{a}}$ & $1.28^{\mathrm{b}}$ & $4.30^{\mathrm{b}}$ \\
\hline & Sids-40 & $5.06^{\mathrm{b}}$ & $64.67^{\mathrm{a}}$ & $28.11^{\mathrm{a}}$ & $14.46^{\mathrm{b}}$ & $3.31^{\mathrm{a}}$ & $4.79^{\mathrm{a}}$ \\
\hline \multirow{2}{*}{ Distances } & $10 \mathrm{~cm}$ & $5.19^{b}$ & $59.48^{\mathrm{b}}$ & $27.58^{\mathrm{a}}$ & $32.74 \mathrm{a}$ & $2.24^{\mathrm{b}}$ & $4.81^{\mathrm{a}}$ \\
\hline & $15 \mathrm{~cm}$ & $5.45^{\mathrm{a}}$ & $63.45^{\mathrm{a}}$ & $25.86^{\mathrm{b}}$ & $32.73 a$ & $2.35^{\mathrm{a}}$ & $4.28^{\mathrm{b}}$ \\
\hline \multirow{4}{*}{$\begin{array}{l}\text { Cropping } \\
\text { system }\end{array}$} & Sole & $5.95^{\mathrm{a}}$ & $70.0^{\mathrm{a}}$ & $30.43^{\mathrm{a}}$ & $33.29 \mathrm{a}$ & $2.76^{\mathrm{a}}$ & $6.33^{\mathrm{a}}$ \\
\hline & One row & $5.58^{b}$ & $64.9^{b}$ & $28.23^{b}$ & $33.03 \mathrm{a}$ & $2.59^{b}$ & $2.28^{\mathrm{d}}$ \\
\hline & Two rows & $5.09^{c}$ & $57.8^{c}$ & $25.14^{\mathrm{c}}$ & $32.77 \mathrm{a}$ & $2.28^{\mathrm{c}}$ & $4.10^{\mathrm{c}}$ \\
\hline & Three rows & $4.67^{\mathrm{d}}$ & $53.1^{\mathrm{d}}$ & $23.09^{d}$ & $31.83 \mathrm{a}$ & $1.55^{\mathrm{d}}$ & $5.48^{\mathrm{b}}$ \\
\hline
\end{tabular}

Means followed by the same letters were not significantly differed according to Duncan's multiple range test.

2-a- Germination percentage and some vegetative growth components:

Data in Table (1) mentioned that all growth parameters as germination percentage of cloves (81.75and 80.15), leaves number per plant (9.48 and 8.62), plant height (72.11 and 70.69) and leaf area (81.14 and 67.06) increased significantly with higher intra-row spacing $(15 \mathrm{~cm})$, respectively in two seasons, while, the lowest values of all the above mentioned traits were obtained from $10 \mathrm{~cm}$ intra-row spacing. Similar results were also reported by Singh et al., (1995). This might be due to the fact that wider row spacing facilitated less competition for space and more availability of light and nutrients to the plants. The increase in growth parameters due to wider spacing in garlic were also reported by Naruka and Dhaka (2001), Alam et al.,(2010), Moravcevic et al (2011) And Olfati et al., (2016).

\section{2-b- Yield and its components:}

Results in table (2), revealed that garlic cultivars, plant spacing and cropping systems effect on garlic yield and its components in seasons 2014/2015 and $2015 / 2016$. Intra-row spacing significantly differed in bulb diameter per plant $(\mathrm{cm})$, fresh and cured bulb weight $(\mathrm{g})$, number of cloves per bulb, clove weight $(\mathrm{g})$ and total yield (ton/fed.). In the present research the yield increased when the lower row spacing were used but the yield improvement i.e bulb diameter, bulb weight, and clove weight, increased when the wider row spacing $(15 \mathrm{~cm})$ was used. Results showed that yield parameters increased at wider row spacing, this might have been due to less number of plants in a given area with low competition for nutrients and sunlight, increasing food assimilatory efficiency and thereby more food reserve in bulbs thereby increasing bulb diameter, fresh weight of bulb and weight of cloves. But, increasing the intra- row spacing, decreased total yield significantly, it was perhaps due to reduction in number of bulbs harvested in a given area. The results obtained are in conformity with the findings of Singh et al., (1995), Muro et al. (2000).Alam et al.,(2010), Moravcevic et al (2011), Olfati et al., (2016) and Azza and Naglaa (2016).

\section{3- Effect of garlic cropping system:}

\section{3- a- Germination percentage and some vegetative growth components:}

Data presented in Table (1) showed that germination $\%$ and all vegetative growth characteristics were significantly affected by cropping systems during the two growing seasons. The highest significant values of germination \% (82.16 and 80.54\%), leaves number per plant (10.5 and 9.6), plant height (79.26 and 77.71) and leaf area (96.44 and 79.70) were recorded when garlic was grown as a sole crop, flowed by garlic cropped with sugar beet at one row (Table 1) during the first and second seasons, respectively. Meanwhile the lowest values of all the above characteristics were recorded when garlic was intercropped with sugar beet on three 
rows. The previous results may be due to competition between plants of garlic and sugar beet for light. The obtained results were harmony with those showed by Abdel Motagally and Metwally (2014), who reflected that, significant decreased was observed by intercropping sugar beet with onion, compared to sole onion.

\section{3- b- Yield and quality:}

Data presented in Table 2 reported that characteristics of garlic yield and its components were significantly affected by intercropping systems, except cloves number per bulb in the two seasons. The previous characteristics gave the highest values when growing garlic in pure stand as sole crop. This may be due to the increase in number of garlic plants compared to intercrop. On the other hand, bulb diameter, fresh bulb weight, cured bulb weight and clove weight surpassed when intercropped one row of garlic on the back of sugar beet compared with the other intercropping systems. On the contrary, intercropping at three rows of garlic on the back of sugar beet gave the highest value compared with the others intercropping systems of total yield per feddan. The reduction in garlic yield in the intercrop associations may be due to the increase in number of garlic plants when compared with pure stand and the severe inter-specific and intra-specific competition between garlic and sugar beet plants, as well as between garlic plants and between sugar beet plants on water and nutrients. Similar results were obtained by Toaima (2006), Ibrahim et al. (2008),Abd El- Zaher et al. (2009) and Abdel Motagally and Metwally (2014).

\section{Interaction effects:}

1- Effect of the interaction between cultivars and intra-row spacing on growth, yield and its components:

Results in tables $3 \& 4$ indicated the effect of interaction between garlic cultivars and intra-row spacing on mean of germination \%, growth, yield and its components. Plant height, leaf area, bulb diameter, fresh bulb weight, curd bulb weight and total yield were significantly affected by the interaction between two varieties and plant spacing in both seasons.

Table 3. Interaction effect between cultivars and intra-row spacing on garlic growth traits

\begin{tabular}{ccccc}
\hline cultivars X Distances & Germination \% & $\begin{array}{c}\text { leaves number } \\
\text { plant }^{-1}\end{array}$ & $\begin{array}{c}\text { Plant } \\
\text { height(cm) }\end{array}$ & $\begin{array}{c}\text { Leaf area } \\
\left(\mathbf{c m}^{2}\right)\end{array}$ \\
\hline & $\mathbf{2 0 1 4 / 2 0 1 5}$ & & & \\
Balady $10 \mathrm{~cm}$ & $82.02^{\mathrm{a}}$ & $8.82^{\mathrm{a}}$ & $71.36^{\mathrm{b}}$ & $70.07^{\mathrm{c}}$ \\
$15 \mathrm{~cm}$ & $82.54^{\mathrm{a}}$ & $9.11^{\mathrm{a}}$ & $76.75^{\mathrm{a}}$ & $80.04^{\mathrm{ab}}$ \\
Sids $-4010 \mathrm{~cm}$ & $80.33^{\mathrm{a}}$ & $9.48^{\mathrm{a}}$ & $65.53^{\mathrm{d}}$ & $77.00^{\mathrm{b}}$ \\
$15 \mathrm{~cm}$ & $80.96^{\mathrm{a}}$ & $9.85^{\mathrm{a}}$ & $67.46^{\mathrm{c}}$ & $82.23^{\mathrm{a}}$ \\
& $\mathbf{2 0 1 5 / 2 0 1 6}$ & & & \\
Balady $10 \mathrm{~cm}$ & $80.41^{\mathrm{a}}$ & $8.02^{\mathrm{a}}$ & $69.96^{\mathrm{b}}$ & $57.91^{\mathrm{c}}$ \\
$15 \mathrm{~cm}$ & $80.92^{\mathrm{a}}$ & $8.28^{\mathrm{a}}$ & $75.25^{\mathrm{a}}$ & $66.15^{\mathrm{ab}}$ \\
Sids $-4010 \mathrm{~cm}$ & $78.75^{\mathrm{a}}$ & $8.62^{\mathrm{a}}$ & $64.24^{\mathrm{d}}$ & $63.63^{\mathrm{b}}$ \\
$15 \mathrm{~cm}$ & $79.38^{\mathrm{a}}$ & $8.96^{\mathrm{a}}$ & $66.14^{\mathrm{c}}$ & $67.96^{\mathrm{a}}$ \\
\hline
\end{tabular}

Means followed by the same letters were not statistically significantly differed according to Duncan's multiple range test.

Table 4. Interaction effect between cultivars and intra-row spacing on garlic yield and quality traits

\begin{tabular}{|c|c|c|c|c|c|c|c|}
\hline \multicolumn{2}{|c|}{ cultivars X Distances } & $\begin{array}{c}\text { Diameter of } \\
\text { bulb }^{-1} \\
(\mathrm{~cm})\end{array}$ & $\begin{array}{c}\text { Fresh bulb } \\
\text { weight } \\
\text { (g) }\end{array}$ & $\begin{array}{c}\text { Cured } \\
\text { bulb } \\
\text { Weight (g) } \\
\end{array}$ & $\begin{array}{l}\text { cloves no. } \\
\text { plant }^{-1}\end{array}$ & $\begin{array}{c}\text { clove } \\
\text { weight }(g)\end{array}$ & $\begin{array}{c}\text { Total } \\
\text { yield } \\
\text { ton/fed. }\end{array}$ \\
\hline \multicolumn{8}{|c|}{$2014 / 2015$} \\
\hline \multirow[t]{2}{*}{ Balady } & $10 \mathrm{~cm}$ & $5.45^{\mathrm{b}}$ & $58.95^{\mathrm{d}}$ & $27.77^{\mathrm{c}}$ & $50.42^{\mathrm{a}}$ & $1.27^{\mathrm{a}}$ & $4.82^{\mathrm{b}}$ \\
\hline & $15 \mathrm{~cm}$ & $5.83^{\mathrm{a}}$ & $61.09^{c}$ & $26.79^{d}$ & $50.56^{\mathrm{a}}$ & $1.32^{\mathrm{a}}$ & $4.03^{\mathrm{c}}$ \\
\hline \multirow[t]{2}{*}{ Sids-40 } & $10 \mathrm{~cm}$ & $5.04^{\mathrm{d}}$ & $63.59^{\mathrm{b}}$ & $31.65^{\mathrm{a}}$ & $14.40^{\mathrm{a}}$ & $3.25^{\mathrm{a}}$ & $5.10^{\mathrm{a}}$ \\
\hline & $15 \mathrm{~cm}$ & $5.18^{\mathrm{c}}$ & $69.62^{\mathrm{a}}$ & $28.91^{\mathrm{b}}$ & $14.23^{\mathrm{a}}$ & $3.44^{\mathrm{a}}$ & $4.77^{\mathrm{b}}$ \\
\hline \multicolumn{8}{|c|}{$2015 / 2016$} \\
\hline \multirow[t]{2}{*}{ Balady } & $10 \mathrm{~cm}$ & $5.40^{\mathrm{b}}$ & $57.23^{\mathrm{c}}$ & $25.78^{c}$ & $50.93^{\mathrm{a}}$ & $1.26^{\mathrm{a}}$ & $4.68^{b}$ \\
\hline & $15 \mathrm{~cm}$ & $5.77^{\mathrm{a}}$ & $59.31^{\mathrm{d}}$ & $24.88^{\mathrm{d}}$ & $51.08^{\mathrm{a}}$ & $1.30^{\mathrm{a}}$ & $3.92^{c}$ \\
\hline \multirow[t]{2}{*}{ Sids -40} & $10 \mathrm{~cm}$ & $4.99^{\mathrm{d}}$ & $61.74^{\mathrm{b}}$ & $26.83^{b}$ & $14.54^{\mathrm{a}}$ & $3.22^{\mathrm{a}}$ & $4.95^{\mathrm{a}}$ \\
\hline & $15 \mathrm{~cm}$ & $5.12^{c}$ & $67.59^{\mathrm{a}}$ & $29.39^{\mathrm{a}}$ & $14.38^{\mathrm{a}}$ & $3.40^{\mathrm{a}}$ & $4.63^{b}$ \\
\hline
\end{tabular}

Means followed by the same letters were not statistically significantly differed according to Duncan's multiple range test 
The highest values of leaf area (82.23 and 67.96), fresh bulb weight (69.62 and 67.59) and curd bulb weight (31.91 and 29.39) resulted from Sids-40 cultivar with $15 \mathrm{~cm}$ intra-row spacing in the two seasons respectively. But the highest values of plant height (76.75and 75.25) as well as bulb diameter (5.83 and 5.7) resulted from Balady cultivar with $15 \mathrm{~cm}$ intra-row spacing in two seasons respectively. Sids-40 cultivar with $10 \mathrm{~cm}$ intra-row spacing gave the highest value of total yield (5.1 and 4.95 ton/fed.) in two seasons respectively

\section{2- Effect of interaction between garlic cultivars and} cropping system on growth, yield and its components:

Interactive effect of cultivars and cropping systems was significant affect for plant height, leaf area, bulb diameter, fresh bulb weight, curd bulb weight, clove weight and total yield in both two seasons (Tables $5 \&$ $6)$.

Within the same garlic cultivar, plant height, leaf area, bulb diameter, fresh bulb weight, curd bulb weight, clove weight and total yield traits of sole crop were usually greater compared with the intercropped stands in two seasons. Within garlic cropping systems, all the previous traits from one row treatment of garlic gave the highest values compared with the others intercropping treatments except total yield. Total yield trait of three rows treatment of garlic gave the highest value compared with the others intercropping treatments.

\section{3- Effect of the interaction between intra-row spacing and cropping system on growth, yield and its components:}

Data in tables $7 \& 8$ indicated the effect of interaction between intra-row spacing and cropping system on garlic growth, yield and its components. Interactive effect of intra-row spacing and cropping systems was significant for plant height, leaf area, bulb diameter, fresh bulb weight, curd bulb weight and total yield in both two seasons (table7\&8). Garlic as sole stand produced plant height, leaf area, bulb diameter, fresh bulb weight, curd bulb weight, clove weight and total yield higher when compared with the intercropped stands in two seasons.

Within intercropping system, one row at $15 \mathrm{~cm}$ intrarow spacing of garlic has the highest values for the previous characteristics except total yield. For total yield trait, three rows at $15 \mathrm{~cm}$ intra-row spacing treatment of garlic gave the highest values compared with the others intercropping treatments.

Table 5. Interaction effect between cultivars and cropping system on garlic growth traits

\begin{tabular}{|c|c|c|c|c|c|}
\hline \multicolumn{2}{|c|}{ Cultivars $x$ cropping } & $\begin{array}{c}\text { Germination } \\
\%\end{array}$ & $\begin{array}{c}\text { leaves no. } \\
\text { plant }^{-1}\end{array}$ & $\begin{array}{l}\text { Plant height } \\
\text { (cm) }\end{array}$ & \multirow[t]{2}{*}{$\begin{array}{c}\text { Leaf area } \\
\left(\mathrm{cm}^{2}\right)\end{array}$} \\
\hline \multicolumn{5}{|c|}{$2014 / 2015$} & \\
\hline \multirow[t]{4}{*}{ Balady } & Sole & $82.96^{\mathrm{a}}$ & $10.01^{\mathrm{a}}$ & $85.29^{\mathrm{a}}$ & $96.56^{\mathrm{a}}$ \\
\hline & One row & $82.60 \mathrm{a}$ & $9.48^{\mathrm{a}}$ & $78.91^{\mathrm{b}}$ & $85.28^{\mathrm{b}}$ \\
\hline & Two rows & $82.30^{\mathrm{a}}$ & $8.57^{\mathrm{a}}$ & $68.91^{\mathrm{d}}$ & $64.62^{\mathrm{d}}$ \\
\hline & Three rows & $81.24^{\mathrm{a}}$ & $7.79^{\mathrm{a}}$ & $63.11^{\mathrm{e}}$ & $53.76^{\mathrm{e}}$ \\
\hline \multirow[t]{4}{*}{ Sids -40} & Sole & $81.35 \mathrm{a}$ & $11.15^{\mathrm{a}}$ & $73.23^{\mathrm{c}}$ & $96.32^{\mathrm{a}}$ \\
\hline & One row & $80.78^{\mathrm{a}}$ & $10.22^{\mathrm{a}}$ & $68.95^{\mathrm{d}}$ & $84.94^{\mathrm{b}}$ \\
\hline & Two rows & $80.54^{\mathrm{a}}$ & $9.08^{\mathrm{a}}$ & $65.06^{\mathrm{e}}$ & $74.93^{\mathrm{c}}$ \\
\hline & Three rows & $79.91^{\mathrm{a}}$ & $8.23^{\mathrm{a}}$ & $58.74^{\mathrm{f}}$ & $62.28^{\mathrm{d}}$ \\
\hline \multicolumn{6}{|c|}{$2015 / 2016$} \\
\hline \multirow[t]{4}{*}{ Balady } & Sole & $81.34^{\mathrm{a}}$ & $9.10^{\mathrm{a}}$ & $83.62^{\mathrm{a}}$ & $79.80^{\mathrm{a}}$ \\
\hline & One row & $80.99^{\mathrm{a}}$ & $8.62^{\mathrm{a}}$ & $77.36^{\mathrm{b}}$ & $70.48^{b}$ \\
\hline & Two rows & $80.69^{a}$ & $7.79^{\mathrm{a}}$ & $67.56^{\mathrm{d}}$ & $53.40^{\mathrm{d}}$ \\
\hline & Three rows & $79.65^{\mathrm{a}}$ & $7.08^{\mathrm{a}}$ & $61.88^{\mathrm{e}}$ & $44.43^{\mathrm{e}}$ \\
\hline \multirow[t]{4}{*}{ Sids -40} & Sole & $79.75^{\mathrm{a}}$ & $10.13^{\mathrm{a}}$ & $71.79^{c}$ & $79.60^{\mathrm{a}}$ \\
\hline & One row & $79.19^{\mathrm{a}}$ & $9.29^{\mathrm{a}}$ & $67.60^{\mathrm{d}}$ & $70.20^{\mathrm{b}}$ \\
\hline & Two rows & $78.96^{\mathrm{a}}$ & $8.25^{\mathrm{a}}$ & $63.78^{\mathrm{e}}$ & $61.92^{\mathrm{c}}$ \\
\hline & Three rows & $78.35^{\mathrm{a}}$ & $7.48^{\mathrm{a}}$ & $57.59^{f}$ & $51.47^{\mathrm{d}}$ \\
\hline
\end{tabular}

Means followed by the same letters were not statistically significantly differed according to Duncan's multiple range test. 
Table 6. Interaction effect between cultivars and cropping system on garlic yield and quality traits

\begin{tabular}{|c|c|c|c|c|c|c|c|}
\hline Cultivar & ntercropping & \multirow[t]{2}{*}{$\begin{array}{l}\text { Diameter of } \\
\text { Bulb }^{-1} \\
(\mathrm{~cm})\end{array}$} & \multirow{2}{*}{$\begin{array}{l}\begin{array}{l}\text { Fresh bulb } \\
\text { weight (g) }\end{array} \\
2014 / 201\end{array}$} & \multirow[t]{2}{*}{$\begin{array}{l}\text { Cured bulb } \\
\text { Weight (g) }\end{array}$} & \multirow[t]{2}{*}{$\begin{array}{l}\text { cloves } \\
\text { no. } \\
\text { plant }^{-1} \\
\end{array}$} & \multirow[t]{2}{*}{$\begin{array}{l}\text { clove } \\
\text { weight } \\
\text { (g) } \\
\end{array}$} & \multirow[t]{2}{*}{$\begin{array}{l}\text { Total } \\
\text { yield } \\
\text { ton/fed }\end{array}$} \\
\hline & & & & & & & \\
\hline \multirow[t]{4}{*}{ Balady } & Sole & $6.44^{\mathrm{a}}$ & $66.3^{\mathrm{c}}$ & $30.13^{c}$ & $51.3^{\mathrm{a}}$ & $1.47^{\mathrm{e}}$ & $6.00^{\mathrm{b}}$ \\
\hline & One row & $5.99^{\mathrm{b}}$ & $62.5^{\mathrm{d}}$ & $28.4^{\mathrm{d}}$ & $50.96^{\mathrm{a}}$ & $1.40^{\mathrm{ef}}$ & $2.21^{\mathrm{f}}$ \\
\hline & Two rows & $5.28^{\mathrm{d}}$ & $58.5^{\mathrm{ef}}$ & $26.6^{\mathrm{ef}}$ & $50.4^{\mathrm{ab}}$ & $1.22^{\mathrm{fg}}$ & $4.14^{\mathrm{d}}$ \\
\hline & Three rows & $4.87^{\mathrm{e}}$ & $52.8^{\mathrm{g}}$ & $24^{\mathrm{g}}$ & $49.3^{\mathrm{a}}$ & $1.09^{\mathrm{g}}$ & $5.35^{\mathrm{c}}$ \\
\hline \multirow[t]{4}{*}{ Sids -40} & Sole & $5.58^{\mathrm{c}}$ & $77.9^{\mathrm{a}}$ & $35.4^{\mathrm{a}}$ & $14.6^{\mathrm{a}}$ & $4.11^{\mathrm{a}}$ & $7.04^{\mathrm{a}}$ \\
\hline & One row & $5.28^{\mathrm{d}}$ & $71.3^{\mathrm{b}}$ & $32.4^{\mathrm{b}}$ & $14.4^{\mathrm{a}}$ & $3.83^{\mathrm{b}}$ & $2.48^{\mathrm{e}}$ \\
\hline & Two rows & $5.01^{\mathrm{e}}$ & $60.6^{\mathrm{de}}$ & $27.56^{\mathrm{de}}$ & $14.5^{\mathrm{a}}$ & $3.40^{\mathrm{c}}$ & $4.29^{\mathrm{d}}$ \\
\hline & Three rows & $4.56^{\mathrm{f}}$ & $56.6^{\mathrm{f}}$ & $25.76^{\mathrm{f}}$ & $13.7^{\mathrm{a}}$ & $2.03^{\mathrm{d}}$ & $5.9^{\mathrm{b}}$ \\
\hline \multicolumn{8}{|c|}{$2015 / 2016$} \\
\hline \multirow[t]{4}{*}{ Balady } & Sole & $6.37^{\mathrm{a}}$ & $64.40^{\mathrm{c}}$ & $28^{\mathrm{c}}$ & $51.83^{\mathrm{a}}$ & $1.46^{\mathrm{e}}$ & $5.82^{\mathrm{b}}$ \\
\hline & One row & $5.93^{\mathrm{b}}$ & $60.66^{\mathrm{d}}$ & $26.37^{\mathrm{d}}$ & $51.48^{\mathrm{a}}$ & $1.38^{\mathrm{ef}}$ & $2.14^{\mathrm{f}}$ \\
\hline & Two rows & $5.23^{\mathrm{d}}$ & $56.79^{\mathrm{ef}}$ & $24.7^{\mathrm{ef}}$ & $50.87^{\mathrm{a}}$ & $1.20^{\mathrm{fg}}$ & $4.02^{\mathrm{d}}$ \\
\hline & Three rows & $4.82^{\mathrm{e}}$ & $51.23^{\mathrm{g}}$ & $22.6^{\mathrm{g}}$ & $49.83^{\mathrm{a}}$ & $1.08^{\mathrm{g}}$ & $5.20^{\mathrm{c}}$ \\
\hline \multirow[t]{4}{*}{ Sids -40} & Sole & $5.53^{\mathrm{c}}$ & $75.64^{\mathrm{a}}$ & $32.86^{\mathrm{a}}$ & $14.75^{\mathrm{a}}$ & $4.07^{\mathrm{a}}$ & $6.83^{\mathrm{a}}$ \\
\hline & One row & $5.23^{\mathrm{d}}$ & $69.19^{\mathrm{b}}$ & $30.08^{b}$ & $14.58^{\mathrm{a}}$ & $3.80^{\mathrm{b}}$ & $2.41^{\mathrm{e}}$ \\
\hline & Two rows & $4.96^{\mathrm{e}}$ & $58.85^{\mathrm{de}}$ & $25.58^{\mathrm{de}}$ & $14.67^{\mathrm{a}}$ & $3.36^{\mathrm{c}}$ & $4.17^{\mathrm{d}}$ \\
\hline & Three rows & $4.51^{\mathrm{f}}$ & $54.98^{\mathrm{f}}$ & $23.912^{\mathrm{f}}$ & $13.83^{\mathrm{a}}$ & $2.01^{\mathrm{d}}$ & $5.76^{\mathrm{b}}$ \\
\hline
\end{tabular}

Means followed by the same letters were not significantly differed according to Duncan's multiple range test.

Table 7. Interaction effect between intra-row spacing and cropping system on garlic growth traits

\begin{tabular}{lcccc}
\hline Distances X cropping system & Germination \% & $\begin{array}{c}\text { leaves no. } \\
\text { plant }\end{array}$ & $\begin{array}{c}\text { Plant height } \\
(\mathbf{c m})\end{array}$ & $\begin{array}{r}\text { Leaf area } \\
\left(\mathbf{c m}^{2}\right)\end{array}$ \\
\hline Garlic sole & \multicolumn{2}{c}{$\mathbf{2 0 1 4 / 2 0 1 5}$} & & \\
Gar.10cm*one row & $82.15 \mathrm{a}$ & $10.6 \mathrm{a}$ & $79.26 \mathrm{a}$ & $96.44 \mathrm{a}$ \\
Gar.10cm*two rows & $81.34 \mathrm{a}$ & $9.54 \mathrm{a}$ & $71.82 \mathrm{c}$ & $80.06 \mathrm{c}$ \\
Gar.10cm*three rows & $81.14 \mathrm{a}$ & $8.64 \mathrm{a}$ & $64.35 \mathrm{~d}$ & $64.15 \mathrm{e}$ \\
Gar.15cm*one row & $80.05 \mathrm{a}$ & $7.83 \mathrm{a}$ & $58.35 \mathrm{e}$ & $53.48 \mathrm{f}$ \\
Gar.15cm*two rows & $82.04 \mathrm{a}$ & $10.1 \mathrm{a}$ & $76.04 \mathrm{~b}$ & $90.16 \mathrm{~b}$ \\
Gar.15cm*three rows & $81.70 \mathrm{a}$ & $9.01 \mathrm{a}$ & $69.62 \mathrm{c}$ & $75.39 \mathrm{~d}$ \\
& $81.11 \mathrm{a}$ & $8.1 \mathrm{a}$ & $63.51 \mathrm{~d}$ & $62.56 \mathrm{e}$ \\
Garlic sole & \multicolumn{2}{c}{$\mathbf{2 0 1 5 / 2 0 1 6}$} & & \\
Gar.10cm*one row & $80.54 \mathrm{a}$ & $9.62 \mathrm{a}$ & $77.71 \mathrm{a}$ & $79.70 \mathrm{a}$ \\
Gar.10cm*two rows & $79.75 \mathrm{a}$ & $8.68 \mathrm{a}$ & $70.42 \mathrm{c}$ & $66.16 \mathrm{c}$ \\
Gar.10cm*three rows & $79.55 \mathrm{a}$ & $7.86 \mathrm{a}$ & $63.08 \mathrm{~d}$ & $53.02 \mathrm{e}$ \\
Gar.15cm*one row & $78.48 \mathrm{a}$ & $7.12 \mathrm{a}$ & $57.20 \mathrm{e}$ & $44.20 \mathrm{f}$ \\
Gar.15cm*two rows & $80.43 \mathrm{a}$ & $9.23 \mathrm{a}$ & $74.55 \mathrm{a}$ & $74.51 \mathrm{~b}$ \\
Gar.15cm*three rows & $80.10 \mathrm{a}$ & $8.19 \mathrm{a}$ & $68.26 \mathrm{c}$ & $62.31 \mathrm{~d}$ \\
\hline
\end{tabular}


Table 8. Interaction effect between intra-row spacing and cropping system on garlic yield and quality traits

\begin{tabular}{|c|c|c|c|c|c|c|}
\hline $\begin{array}{c}\text { Distances } X \\
\text { Cropping system }\end{array}$ & $\begin{array}{l}\text { Diameter } \\
\text { Of Bulb }^{-1} \\
\text { (cm) }\end{array}$ & $\begin{array}{l}\text { Fresh bulb } \\
\text { weight(g) }\end{array}$ & $\begin{array}{l}\text { Cured } \\
\text { bulb } \\
\text { Weight } \\
\text { (g) }\end{array}$ & $\begin{array}{l}\text { Cloves no. } \\
\text { plant }^{-1}\end{array}$ & $\begin{array}{l}\text { Clove } \\
\text { weight(g) }\end{array}$ & $\begin{array}{l}\text { Total yield } \\
\text { (ton/fed.) }\end{array}$ \\
\hline
\end{tabular}

\begin{tabular}{|c|c|c|c|c|c|c|}
\hline \multicolumn{7}{|c|}{ 2014/2015 } \\
\hline Garlic sole & $6.01 \mathrm{a}$ & $72.12 \mathrm{a}$ & $32.76^{\mathrm{a}}$ & $33.17 \mathrm{a}$ & $2.79 \mathrm{a}$ & $6.52 \mathrm{a}$ \\
\hline Gar. $10 \mathrm{~cm} *$ one row & $5.48 \mathrm{c}$ & $64.66 \mathrm{c}$ & $29.38^{\mathrm{c}}$ & $33.08 \mathrm{a}$ & $2.57 \mathrm{a}$ & $2.51 \mathrm{f}$ \\
\hline Gar.10cm*two rows & $4.96 \mathrm{~d}$ & $56.24 \mathrm{~d}$ & $25.58^{\mathrm{d}}$ & $32.04 \mathrm{a}$ & $2.14 \mathrm{a}$ & $4.53 c$ \\
\hline Gar.10cm*three rows & $4.53 \mathrm{e}$ & $52.06 \mathrm{e}$ & $23.68^{\mathrm{e}}$ & $31.35 \mathrm{a}$ & $1.54 \mathrm{a}$ & $6.26 \mathrm{~b}$ \\
\hline Gar.15cm*one row & $5.78 b$ & $69.08 \mathrm{~b}$ & $31.42^{\mathrm{b}}$ & $32.32 \mathrm{a}$ & $2.66 \mathrm{a}$ & $2.17 \mathrm{~g}$ \\
\hline Gar. $15 \mathrm{~cm} *$ two rows & $5.33 \mathrm{c}$ & $62.88 \mathrm{c}$ & $28.58^{\mathrm{c}}$ & $32.84 \mathrm{a}$ & $2.47 \mathrm{a}$ & $3.90 \mathrm{e}$ \\
\hline Gar. $15 \mathrm{~cm} *$ three rows & $4.90 \mathrm{~d}$ & $57.34 \mathrm{~d}$ & $26.08^{\mathrm{d}}$ & $31.68 \mathrm{a}$ & $1.59 \mathrm{a}$ & $5.02 \mathrm{c}$ \\
\hline \multicolumn{7}{|c|}{$2015 / 2016$} \\
\hline Garlic sole & $5.95 \mathrm{a}$ & $70.02 \mathrm{a}$ & $30.4^{\mathrm{a}}$ & $33.50 \mathrm{a}$ & $2.76 \mathrm{a}$ & $6.33 a$ \\
\hline Gar. $10 \mathrm{~cm} *$ one row & $5.43 \mathrm{c}$ & $62.78 \mathrm{c}$ & $27.28^{\mathrm{c}}$ & $33.42 \mathrm{a}$ & $2.55 \mathrm{a}$ & $2.44 \mathrm{f}$ \\
\hline Gar. $10 \mathrm{~cm} *$ two rows & $4.91 \mathrm{~d}$ & $54.60 \mathrm{~d}$ & $23.73^{\mathrm{d}}$ & $32.37 \mathrm{a}$ & $2.12 \mathrm{a}$ & $4.40 \mathrm{c}$ \\
\hline Gar. $10 \mathrm{~cm} *$ three rows & $4.49 \mathrm{e}$ & $50.54 \mathrm{e}$ & $21.98^{\mathrm{e}}$ & $31.67 \mathrm{a}$ & $1.52 \mathrm{a}$ & $6.08 \mathrm{~b}$ \\
\hline Gar. $15 \mathrm{~cm} *$ one row & $5.72 b$ & $67.07 \mathrm{~b}$ & $29.17^{\mathrm{b}}$ & $32.65 \mathrm{a}$ & $2.63 \mathrm{a}$ & $2.11 \mathrm{~g}$ \\
\hline Gar. $15 \mathrm{~cm} *$ two rows & $5.28 \mathrm{c}$ & $61.04 \mathrm{c}$ & $26.56^{\mathrm{c}}$ & $33.17 \mathrm{a}$ & $2.44 a$ & $3.79 \mathrm{e}$ \\
\hline Gar. $15 \mathrm{~cm} *$ three rows & $4.85 \mathrm{~d}$ & $55.67 \mathrm{~d}$ & $24.2^{\mathrm{d}}$ & $32.00 \mathrm{a}$ & $1.57 \mathrm{a}$ & $4.88 \mathrm{c}$ \\
\hline
\end{tabular}

Means followed by the same letters were not statistical significantly differed according to Duncan's multiple range

\section{4- Effect of interaction between garlic cultivars, intra-row spacing and cropping system on garlic growth, yield and its components:}

Data present in tables 9 and 10 indicated the effect of interaction between garlic cultivars, intra-row spacing and cropping system on garlic germination \%, growth, yield and its components in two seasons. Generally, garlic as sole stand has the highest values for all characteristics in two seasons. The highest values of plant height (80.98 and 79.39) as well as bulb diameter (6.13 and 6.00) in two seasons respectively resulted from Balady cv. planted at 15 intra-row spacing with one row. Meanwhile, Sids- $40 \mathrm{cv}$. planted at $15 \mathrm{~cm}$ intrarow spacing with one row gave the highest values of fresh bulb weight (73.39 and 71.25), curd bulb weight (33.36 and 31.00) in two seasons respectively. Sids-40 cv. $x 15 \mathrm{~cm}$ intra-row spacing $\mathrm{x}$ three rows have the highest value for total yield (6.26 and 6.08) respectively in two seasons. The previous results may be due to the intra specific competition between garlic plants, as well as inter competition between garlic plants and sugar beet plants for light, water, solar radiation, moisture and nutrients (Masri and Safina 2015).

*Performance of sugar beet:-

\section{Main effects:}

Data presented in Tables 11 \&12 showed the effected of cultivars, intra- row spacing and cropping system of garlic on sugar beet traits in 2014/2015 and 2015/2016 seasons.

\section{1- Effect of garlic cultivars on sugar beet quality:}

The results revealed that there are insignificant effects on leaves number, leaves weight ( $\mathrm{g})$, root weight $(\mathrm{g})$, root length $(\mathrm{cm})$, yield weight (ton/fed.), sucrose percentage and purity percentage in both seasons. Meanwhile, garlic cultivars gave significant effect on root diameter in two seasons. Balady cv. gave the highest value of root diameter (23.06 and 21.67) in both seasons respectively. There results agreed with those reported by Hussein and Metwally (2012), El-Shamy et al. (2015).

\section{2- Effect of garlic plant spacing on sugar beet quality:}

Sugar beet was significantly influenced by the different plant spacing of garlic intercropped with sugar beet in two seasons.

Intra-row spacing at $15 \mathrm{~cm}$ gave the highest values of leaves number (31.04 and 28.54), leaves weight (296.3 and 297.6), root weight (702.1 and 665.8), root diameter (22.60 and 21.21), yield weight (25.43 and 24.64 ton/fed.), sucrose (16.34and $16.13 \%$ ) and purity (68.63 and $68.15 \%)$ in two seasons respectively. On contrary, root length trait of sugar beet increased with decreased the intra-row spacing, as $10 \mathrm{~cm}$ intra -row spacing gave the highest values in two seasons (16.92 and $16.08 \mathrm{~cm}$ ) respectively.). These results are in accordance with those obtained by Abdel Motagally and Metwally (2014). 
3- Effect of garlic cropping system on sugar beet quality:

The data of leaves number, leaves weight, root weight, root diameter, root length, yield weight (ton/fed.), sucrose $\%$ and purity $\%$ of sugar beet differed significantly due to row proportion of garlic intercropped with sugar beet in two seasons.

Sole sugar beet recorded significantly higher leaves number (33.00and 30.33), leaves weight (383.3 and $358.3)$, root weight(833.3 and 800.0), root diameter(26.73 and25.3), yield weight (29.00 and 28.16 ton/fed.), sucrose (16.8 and $16.6 \%$ ) and purity ( 73.38 and $72.7 \%$ ) respectively in two seasons compared to other treatments. But, sole sugar beet recorded Table 9. Interaction effect between garlic cultivars, intra-row spacing and cropping system on garlic growth traits

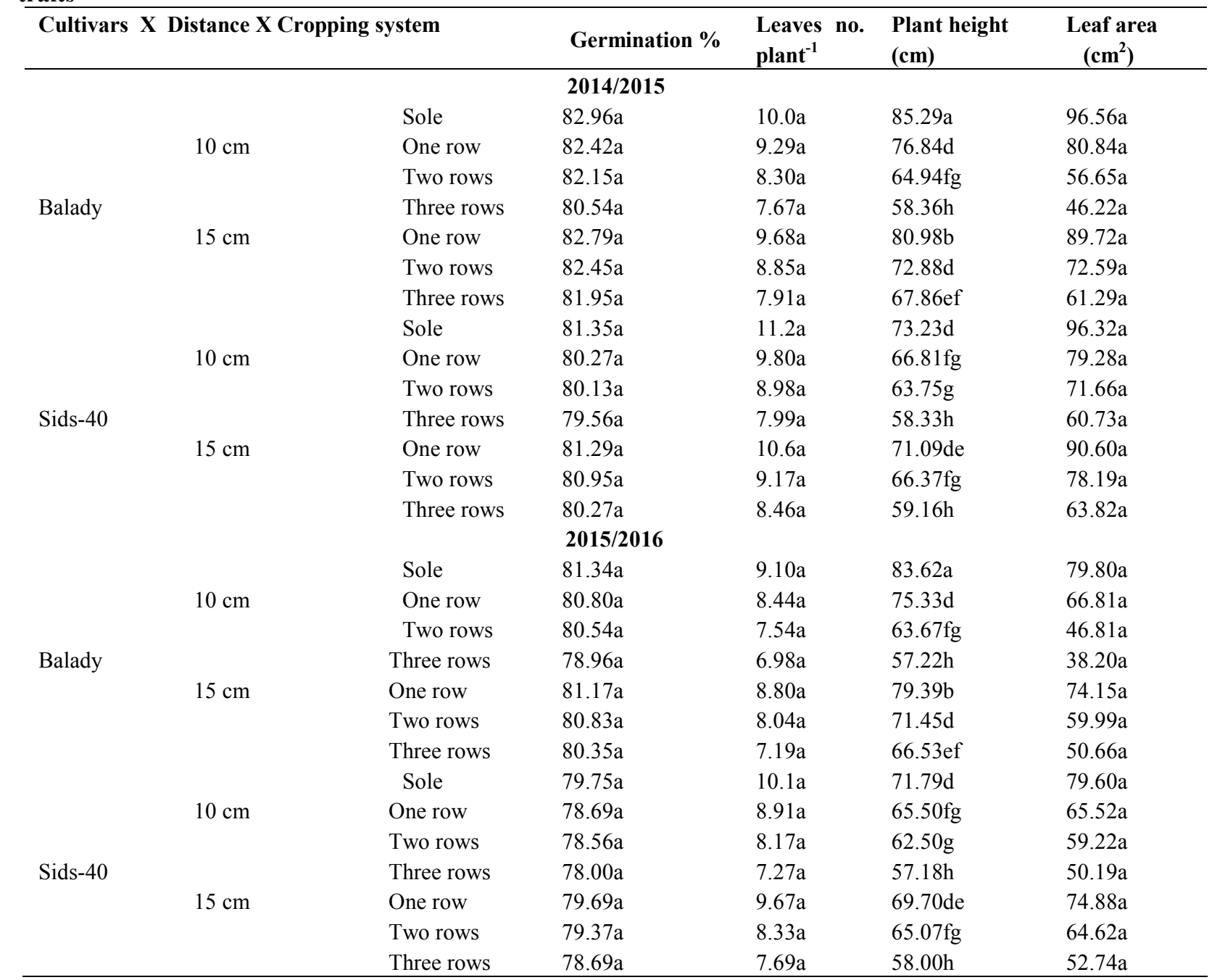

Means followed by the same letters were not statistical significantly differed according to Duncan's multiple range test. significantly lower root length compared to the other treatments. In general, increase in the row ratios of garlic resulted in gradual reduction of sugar beet characteristics except root length parameter. Growing of garlic in one row recorded significantly higher for all characteristics than two and three row. And lower sugar beet for all characteristics were recorded under three row of garlic. On the contrary, significantly lower root length was recorded by sugar beet growing with three row of garlic in two seasons $(15.5$ and $14.5 \mathrm{~cm}$ respectively) compared to other treatments. These results are in a great agreement with those obtained by Hussein and Metwally (2012), Abou Khadra et al. (2013) and Abdel Motagally and Metwally (2014). 
Table 10. Interaction effect between garlic cultivars, intra-row spacing and cropping system on garlic yield and quality traits

\begin{tabular}{|c|c|c|c|c|c|c|c|c|}
\hline Cultivars & $\begin{array}{c}\text { X Distances } \\
\text { system }\end{array}$ & X Cropping & $\begin{array}{c}\text { Bulb } \\
\text { Diameter } \\
\text { (cm) }\end{array}$ & $\begin{array}{c}\text { Fresh } \\
\text { bulb } \\
\text { weight } \\
\text { (g) } \\
\end{array}$ & $\begin{array}{c}\text { Cured } \\
\text { bulb } \\
\text { Weight } \\
(\mathrm{g}) \\
\end{array}$ & $\begin{array}{c}\text { cloves no. } \\
\text { plant }^{-1}\end{array}$ & $\begin{array}{c}\text { clove } \\
\text { weight } \\
\text { (g) }\end{array}$ & $\begin{array}{c}\text { Total } \\
\text { yield } \\
\text { ton/fed }\end{array}$ \\
\hline \multicolumn{9}{|c|}{$2014 / 2015$} \\
\hline \multirow{6}{*}{ Balady } & & Sole & $6.44 \mathrm{a}$ & $66.33 \mathrm{~cd}$ & $30.13 \mathrm{~cd}$ & $51.5 \mathrm{a}$ & $1.47 \mathrm{a}$ & $6.00 \mathrm{~b}$ \\
\hline & $10 \mathrm{~cm}$ & One row & $5.84 \mathrm{c}$ & $60.17 \mathrm{fg}$ & $27.33 \mathrm{fg}$ & $51.80 \mathrm{a}$ & $1.37 \mathrm{a}$ & $2.39 \mathrm{~g}$ \\
\hline & & Two rows & $5.00 \mathrm{fg}$ & $57.24 \mathrm{gh}$ & $26.03 \mathrm{gh}$ & $49.90 \mathrm{a}$ & $1.17 \mathrm{a}$ & $4.62 \mathrm{~d}$ \\
\hline & & Three rows & $4.53 \mathrm{~h}$ & $52.04 \mathrm{~h}$ & $23.66 \mathrm{i}$ & $48.50 \mathrm{a}$ & $1.08 \mathrm{a}$ & $6.26 \mathrm{~b}$ \\
\hline & $15 \mathrm{~cm}$ & One row & $6.13 b$ & $64.78 \mathrm{de}$ & $29.46 \mathrm{de}$ & $50.13 a$ & $1.43 \mathrm{a}$ & $2.03 \mathrm{~h}$ \\
\hline & & Two rows & $5.56 \mathrm{~d}$ & $59.75 \mathrm{fg}$ & $27.16 \mathrm{fg}$ & $50.80 \mathrm{a}$ & $1.26 \mathrm{a}$ & $3.66 \mathrm{f}$ \\
\hline \multirow{8}{*}{ Sids-40 } & & Three rows & $5.20 \mathrm{ef}$ & $53.49 \mathrm{~h}$ & 24.33hi & $50.16 \mathrm{a}$ & $1.10 \mathrm{a}$ & $4.45 \mathrm{ge}$ \\
\hline & & Sole & $5.58 \mathrm{~cd}$ & $77.9 \mathrm{a}$ & $35.4 \mathrm{a}$ & $14.9 \mathrm{a}$ & $4.11 \mathrm{a}$ & $7.04 \mathrm{a}$ \\
\hline & $10 \mathrm{~cm}$ & One row & $5.13 \mathrm{fg}$ & $69.15 \mathrm{c}$ & $31.4 \mathrm{c}$ & $14.4 \mathrm{a}$ & $3.78 \mathrm{a}$ & $2.64 \mathrm{~g}$ \\
\hline & & Two rows & $4.91 \mathrm{~g}$ & $55.23 \mathrm{~h}$ & $25.13 \mathrm{hi}$ & $14.2 \mathrm{a}$ & $3.11 \mathrm{a}$ & $4.45 \mathrm{de}$ \\
\hline & & Three rows & $4.53 \mathrm{~h}$ & $52.08 \mathrm{i}$ & $23.7 \mathrm{i}$ & $14.2 \mathrm{a}$ & $1.99 \mathrm{a}$ & $6.27 \mathrm{~b}$ \\
\hline & $15 \mathrm{~cm}$ & One row & $5.43 \mathrm{de}$ & $73.39 b$ & $33.36 \mathrm{~b}$ & $14.5 \mathrm{a}$ & $3.9 \mathrm{a}$ & $2.32 \mathrm{gh}$ \\
\hline & & Two rows & $5.10 \mathrm{fg}$ & $66.00 \mathrm{~cd}$ & $30 \mathrm{~cd}$ & $14.9 \mathrm{a}$ & $3.68 \mathrm{a}$ & $4.14 \mathrm{e}$ \\
\hline & & Three rows & $4.59 \mathrm{~h}$ & $61.19 \mathrm{ef}$ & $27.83 \mathrm{ef}$ & $13.2 \mathrm{a}$ & 2.08 & $5.60 \mathrm{c}$ \\
\hline \multicolumn{9}{|c|}{$2015 / 2016$} \\
\hline \multirow{7}{*}{ Balady } & & Sole & $6.37 \mathrm{a}$ & $64.4 \mathrm{~cd}$ & $28 \mathrm{~cd}$ & $52.00 \mathrm{a}$ & $1.46 \mathrm{a}$ & $5.82 \mathrm{~b}$ \\
\hline & $10 \mathrm{~cm}$ & One row & $5.7 \mathrm{c}$ & $58.4 \mathrm{fg}$ & $25.4 \mathrm{fg}$ & $52.33 \mathrm{a}$ & $1.35 \mathrm{a}$ & $2.32 \mathrm{~g}$ \\
\hline & & Two rows & $8.95 f g$ & $55.6 \mathrm{gh}$ & $24.16 \mathrm{~h}$ & $50.40 \mathrm{a}$ & $1.16 \mathrm{a}$ & $4.49 \mathrm{~d}$ \\
\hline & & Three rows & $4.49 \mathrm{~h}$ & $50.5 \mathrm{~h}$ & $21.96 \mathrm{i}$ & $49.00 \mathrm{a}$ & $1.07 \mathrm{a}$ & $6.08 \mathrm{~b}$ \\
\hline & $15 \mathrm{~cm}$ & One row & $6.07 \mathrm{~b}$ & $62.9 \mathrm{de}$ & $27.33 \mathrm{de}$ & $50.63 a$ & 1.42 & $1.97 \mathrm{~h}$ \\
\hline & & Two rows & $5.50 \mathrm{~d}$ & $58.0 \mathrm{fg}$ & $25.23 \mathrm{fg}$ & $51.33 \mathrm{a}$ & $1.25 \mathrm{a}$ & $3.56 \mathrm{f}$ \\
\hline & & Three rows & $5.15 \mathrm{ef}$ & $51.9 \mathrm{~h}$ & 22.56hi & $50.67 \mathrm{a}$ & $1.09 \mathrm{a}$ & $4.32 \mathrm{ge}$ \\
\hline \multirow{7}{*}{ Sids-40 } & & Sole & $5.53 \mathrm{~cd}$ & $75.64 \mathrm{a}$ & $32.86 a$ & $14.50 \mathrm{a}$ & $4.07 \mathrm{a}$ & $6.83 \mathrm{a}$ \\
\hline & $10 \mathrm{~cm}$ & One row & $5.08 \mathrm{fg}$ & $67.13 \mathrm{c}$ & $29.16 \mathrm{c}$ & $14.50 \mathrm{a}$ & $3.74 \mathrm{a}$ & $2.56 \mathrm{~g}$ \\
\hline & & Two rows & $4.86 \mathrm{~g}$ & $53.62 \mathrm{~h}$ & 23.3hi & $14.33 \mathrm{a}$ & $3.08 \mathrm{a}$ & $4.32 \mathrm{de}$ \\
\hline & & Three rows & $4.48 \mathrm{~h}$ & $50.56 \mathrm{i}$ & $22.0 \mathrm{i}$ & $14.33 \mathrm{a}$ & $1.97 \mathrm{a}$ & $6.08 \mathrm{~b}$ \\
\hline & $15 \mathrm{~cm}$ & One row & $5.38 \mathrm{de}$ & $71.25 \mathrm{~b}$ & $31.0 \mathrm{~b}$ & $14.67 \mathrm{a}$ & $3.85 \mathrm{a}$ & $2.25 \mathrm{gh}$ \\
\hline & & Two rows & $5.05 \mathrm{fg}$ & $64.07 \mathrm{~cd}$ & $27.86 \mathrm{~cd}$ & $15.00 \mathrm{a}$ & $3.64 \mathrm{a}$ & $4.02 \mathrm{e}$ \\
\hline & & Three rows & $4.54 \mathrm{~h}$ & $59.41 \mathrm{e}$ & $25.83 \mathrm{ef}$ & $13.33 \mathrm{a}$ & $2.06 \mathrm{a}$ & $5.43 \mathrm{c}$ \\
\hline
\end{tabular}


Table 11. Mean effect of garlic cultivars, plant spacing and cropping system on sugar beet growth components

\begin{tabular}{|c|c|c|c|c|c|}
\hline \multicolumn{2}{|c|}{ Treatments } & \multirow{3}{*}{$\begin{array}{c}\begin{array}{c}\text { Leaves } \\
\text { number plant }\end{array} \\
30.08^{\mathrm{a}}\end{array}$} & \multirow{2}{*}{$\begin{array}{c}\text { Leaves } \\
\text { weight plant }^{-1} \\
2014 / 2015\end{array}$} & \multirow{3}{*}{$\begin{array}{c}\begin{array}{c}\text { Root weight } \\
\text { plant }^{-1}\end{array} \\
679.2^{\mathrm{a}}\end{array}$} & \multirow{2}{*}{ Root diameter } \\
\hline & & & & & \\
\hline \multirow[t]{2}{*}{ cultivars } & Balady & & $291.7^{\mathrm{a}}$ & & $22.67^{\mathrm{a}}$ \\
\hline & Sids- -40 & $30.25^{\mathrm{a}}$ & $273.3^{\mathrm{a}}$ & $670.8^{\mathrm{a}}$ & $21.71^{\mathrm{a}}$ \\
\hline \multirow[t]{2}{*}{ Distances } & $10 \mathrm{~cm}$ & $29.29^{\mathrm{a}}$ & $268.8^{\mathrm{b}}$ & $647.9^{\mathrm{b}}$ & $21.67^{\mathrm{b}}$ \\
\hline & $15 \mathrm{~cm}$ & $31.04^{\mathrm{b}}$ & $296.3^{\mathrm{a}}$ & $702.1^{\mathrm{a}}$ & $22.71^{\mathrm{a}}$ \\
\hline \multirow{4}{*}{$\begin{array}{l}\text { Cropping } \\
\text { system }\end{array}$} & Sole & $33.00^{\mathrm{a}}$ & $383.3^{\mathrm{a}}$ & $833.3^{\mathrm{d}}$ & $26.00^{\mathrm{a}}$ \\
\hline & One row & $31.75^{\mathrm{b}}$ & $317.5^{\mathrm{b}}$ & $754.2^{\mathrm{e}}$ & $23.75^{\mathrm{b}}$ \\
\hline & Two rows & $28.83^{\mathrm{c}}$ & $237.5^{\mathrm{c}}$ & $645.8^{f}$ & $21.75^{\mathrm{c}}$ \\
\hline & Three rows & $27.08^{d}$ & $191.7^{\mathrm{d}}$ & $466.7^{\mathrm{g}}$ & $17.25^{\mathrm{d}}$ \\
\hline & & & 2015/2016 & & \\
\hline \multirow[t]{2}{*}{ cultivars } & Balady & $28.00 \mathrm{a}$ & $275.3 \mathrm{a}$ & $645.8 \mathrm{a}$ & $21.67 \mathrm{a}$ \\
\hline & Sids- 40 & $27.58 \mathrm{a}$ & $275.1 \mathrm{a}$ & $632.8 \mathrm{a}$ & $20.13 \mathrm{a}$ \\
\hline \multirow[t]{2}{*}{ Distances } & $10 \mathrm{~cm}$ & $27.04 \mathrm{a}$ & $252.8 \mathrm{~b}$ & $612.9 \mathrm{~b}$ & $20.58 b$ \\
\hline & $15 \mathrm{~cm}$ & $28.54 \mathrm{~b}$ & $297.6 \mathrm{a}$ & $665.8 \mathrm{c}$ & $21.21 \mathrm{a}$ \\
\hline \multirow{4}{*}{$\begin{array}{l}\text { Cropping } \\
\text { system }\end{array}$} & Sole & $30.33 a$ & $358.3 \mathrm{a}$ & $800.0 \mathrm{~d}$ & $25.3 \mathrm{a}$ \\
\hline & One row & $28.80 \mathrm{~b}$ & $324.1 \mathrm{~b}$ & $700.4 \mathrm{e}$ & $21.8 \mathrm{~b}$ \\
\hline & Two rows & $26.92 \mathrm{c}$ & $240.4 c$ & $620.8 \mathrm{f}$ & $20.1 \mathrm{c}$ \\
\hline & Three rows & $25.08 \mathrm{~d}$ & $177.9 \mathrm{~d}$ & $436.0 \mathrm{~g}$ & $16.3 d$ \\
\hline
\end{tabular}

Means followed by the same letters were not statistical significantly differed according to Duncan's multiple range

Table 12. Effect of garlic cultivars, plant spacing and cropping system on sugar beet quality

\begin{tabular}{llcccc}
\multicolumn{2}{c}{ Treatments } & $\begin{array}{c}\text { Root length } \\
\text { (cm) }\end{array}$ & $\begin{array}{l}\text { Yield weight fad } \\
\text { (ton) }\end{array}$ & $\begin{array}{l}\text { Sucrose } \\
\text { \% }\end{array}$ & $\begin{array}{l}\text { Purity } \\
\%\end{array}$ \\
\hline \multirow{3}{*}{ cultivars } & Balady & $15.88^{\mathrm{a}}$ & $\mathbf{2 0 1 4 / 2 0 1 5}$ & & \\
& Sistances & $15.79^{\mathrm{a}}$ & $24.93^{\mathrm{a}}$ & $16.10^{\mathrm{a}}$ & $68.68^{\mathrm{a}}$ \\
& Sids-40 & $16.92^{\mathrm{a}}$ & $24.56^{\mathrm{a}}$ & $15.94^{\mathrm{a}}$ & $67.70^{\mathrm{a}}$ \\
Cropping & $10 \mathrm{~cm}$ & $14.75^{\mathrm{a}}$ & $25.43^{\mathrm{a}}$ & $15.69^{\mathrm{a}}$ & $67.74^{\mathrm{b}}$ \\
system & $15 \mathrm{~cm}$ & $15.33^{\mathrm{bc}}$ & $29.00^{\mathrm{c}}$ & $16.34^{\mathrm{a}}$ & $68.63^{\mathrm{a}}$ \\
& Sole & $14.25^{\mathrm{bc}}$ & $25.07^{\mathrm{c}}$ & $16.80^{\mathrm{b}}$ & $73.38^{\mathrm{a}}$ \\
& One row & $15.67^{\mathrm{cd}}$ & $23.88^{\mathrm{d}}$ & $16.52^{\mathrm{b}}$ & $68.71^{\mathrm{b}}$ \\
& Two rows & $18.08^{\mathrm{d}}$ & $22.02^{\mathrm{d}}$ & $15.71^{\mathrm{c}}$ & $66.21^{\mathrm{c}}$ \\
cultivars & Three rows & & $\mathbf{2 0 1 5 / 2 0 1 6}$ & $15.04^{\mathrm{c}}$ & $64.44^{\mathrm{d}}$ \\
& & & $24.34 \mathrm{a}$ & $15.83 \mathrm{a}$ & $67.97 \mathrm{a}$ \\
Distances & Balady & $14.88 \mathrm{a}$ & $24.30 \mathrm{a}$ & $15.71 \mathrm{a}$ & $67.28 \mathrm{a}$ \\
Cropping & Sids-40 & $15.17 \mathrm{a}$ & $23.99 \mathrm{a}$ & $15.41 \mathrm{a}$ & $67.08 \mathrm{~b}$ \\
system & $10 \mathrm{~cm}$ & $16.08 \mathrm{a}$ & $24.64 \mathrm{a}$ & $16.13 \mathrm{a}$ & $68.15 \mathrm{a}$ \\
& $15 \mathrm{~cm}$ & $13.96 \mathrm{a}$ & $28.16 \mathrm{c}$ & $16.60 \mathrm{~b}$ & $72.70 \mathrm{a}$ \\
& Sole & $14.00 \mathrm{bc}$ & $24.36 \mathrm{c}$ & $16.12 \mathrm{~b}$ & $67.89 \mathrm{~b}$ \\
& One row & $14.50 \mathrm{bc}$ & $23.26 \mathrm{~d}$ & $15.49 \mathrm{c}$ & $65.79 \mathrm{c}$ \\
\hline
\end{tabular}

Means followed by the same letters were not significantly differed according to Duncan's multiple range test

\section{Interaction effects:}

1- Effect of the interaction between varieties and intra-row spacing on sugar beet quality:

The results in Tables 13 and 14 indicated that the effect of interaction between cultivars and intra-row spacing of garlic on sugar beet growth, yield and its components in 2014/2015 and $2015 / 2016$. There were insignificant effects on all growth, yield and its components except, total yield / fed. and purity in two seasons. Balady cv. at $15 \mathrm{~cm}$ intra -row spacing was recorded higher for yield weight (25.48 and 25.08) and purity \% (69.97 and 68.98) compared with the other treatments in two seasons respectively irrespective of various row proportion tested. 
2- Effect of the interaction between garlic cultivars and cropping system on sugar beet quality:

The results in Tables 15 and 16 showed that the effect of interaction between cultivars and cropping systems of garlic on sugar beet growth, yield and its components in 2014/2015and $2015 / 2016$. There were no significant effects on all growth, yield and its components in two seasons except, leaves number and leaves weight were significantly influenced in $2^{\text {nd }}$ seasons also, root diameter/plant were significantly influenced in two seasons. Sole sugar beet recorded higher leaves number (30.33), leaves weight per plant (358.3) and root diameter (2673 and 25.33) compared with the other treatments. While, sugar beet gave the highest values when intercropped with garlic at one row irrespective of different intra spacing examined.
3- Effect of the interaction between intra-row spacing and cropping system on sugar beet quality:

The data on leaves number per plant, leaves weight per plant, root weight, root diameter, root length, yield weight per fed. sucrose $\%$ and purity $\%$ of sugar beet differed significantly due to interaction between intra row spacing and cropping systems of garlic (Table17 and 18).

Sole sugar beet recorded significantly higher values of all growth, yield and its components (leaves number, leaves weight, root weight, root diameter, yield weight per fed. and purity \%) except, root length and sucrose $\%$ compared to other treatments. However, growing of sugar beet intercropped with garlic at $15 \mathrm{~cm}$ intra-row spacing one garlic row recorded higher values for leaves number(33 and 30.17), leaves weight(370.8 and $345)$, root weight( 800 and 756.67$)$, root diameter( 24.33 and 22.17), yield weight (25.65 and 24.82), sucrose(17.04 and 16.77\%) and purity (69.62 and $68.64 \%$ ) respectively in two seasons.

Table 13. Interaction Effect between cultivars and intra-row spacing on sugar beet growth traits

\begin{tabular}{|c|c|c|c|c|c|}
\hline & cultivars $X$ Distances & $\begin{array}{c}\text { Leave number } \\
\text { plant }^{-1}\end{array}$ & $\begin{array}{c}\text { Leave weight } \\
\text { plant }^{-1}\end{array}$ & $\begin{array}{c}\text { Root weight } \\
\text { plant }^{-1} \\
\end{array}$ & $\begin{array}{c}\text { Root diameter } \\
\text { plant }^{-1}\end{array}$ \\
\hline \multicolumn{6}{|c|}{$2014 / 2015$} \\
\hline \multirow[t]{2}{*}{ Balady } & $10 \mathrm{~cm}$ & $29.33^{\mathrm{a}}$ & $266.67^{\mathrm{a}}$ & $650.00^{\mathrm{a}}$ & $22.65^{\mathrm{a}}$ \\
\hline & $15 \mathrm{~cm}$ & $30.83^{\mathrm{a}}$ & $316.67^{\mathrm{a}}$ & $708.33^{\mathrm{a}}$ & $23.32^{\mathrm{a}}$ \\
\hline \multirow[t]{2}{*}{ Sids-40 } & $10 \mathrm{~cm}$ & $29.25^{\mathrm{a}}$ & $270.83^{\mathrm{a}}$ & $645.83^{\mathrm{a}}$ & $21.32^{\mathrm{a}}$ \\
\hline & $15 \mathrm{~cm}$ & $31.25^{\mathrm{a}}$ & $320.8^{\mathrm{a}}$ & $695.83^{\mathrm{a}}$ & $21.73^{\mathrm{a}}$ \\
\hline \multicolumn{6}{|c|}{$2015 / 2016$} \\
\hline \multirow[t]{2}{*}{ Balady } & $10 \mathrm{~cm}$ & $27.08^{\mathrm{a}}$ & $256.25^{\mathrm{a}}$ & $619.75^{\mathrm{a}}$ & $21.25^{\mathrm{a}}$ \\
\hline & $15 \mathrm{~cm}$ & $28.92^{\mathrm{a}}$ & $294.33^{\mathrm{a}}$ & $671.92^{\mathrm{a}}$ & $22.08^{\mathrm{a}}$ \\
\hline \multirow[t]{2}{*}{ Sids-40 } & $10 \mathrm{~cm}$ & $27.00^{\mathrm{a}}$ & $249.33^{\mathrm{a}}$ & $606.00^{\mathrm{a}}$ & $19.92^{\mathrm{a}}$ \\
\hline & $15 \mathrm{~cm}$ & $28.17^{\mathrm{a}}$ & $300.83^{\mathrm{a}}$ & $659.58^{\mathrm{a}}$ & $20.33^{\mathrm{a}}$ \\
\hline
\end{tabular}

Means followed by the same letters were not statistical significantly differed according to Duncan's multiple range test.

Table 14. Interaction Effect between cultivars and intra-row spacing on sugar beet yield quality traits

\begin{tabular}{|c|c|c|c|c|c|}
\hline & Cultivars X Distances & root length $(\mathrm{cm})$ & $\begin{array}{c}\text { yield } \\
\text { weight(ton) }\end{array}$ & Sucrose\% & Purity \% \\
\hline \multicolumn{6}{|c|}{$2014 / 2015$} \\
\hline \multirow[t]{2}{*}{ Balady } & $10 \mathrm{~cm}$ & $16.73^{\mathrm{a}}$ & $24.38^{\mathrm{b}}$ & $15.77^{\mathrm{a}}$ & $67.91^{\mathrm{b}}$ \\
\hline & $15 \mathrm{~cm}$ & $14.00^{\mathrm{a}}$ & $25.48^{\mathrm{a}}$ & $16.42^{\mathrm{a}}$ & $69.97^{\mathrm{a}}$ \\
\hline \multirow[t]{2}{*}{ Sids-40 } & $10 \mathrm{~cm}$ & $17.93^{\mathrm{a}}$ & $24.73^{b}$ & $15.61^{\mathrm{a}}$ & $68.24^{\mathrm{b}}$ \\
\hline & $15 \mathrm{~cm}$ & $14.89^{\mathrm{a}}$ & $25.39^{\mathrm{a}}$ & $16.26^{\mathrm{a}}$ & $68.31^{\mathrm{b}}$ \\
\hline \multicolumn{6}{|c|}{$2015 / 2016$} \\
\hline \multirow[t]{2}{*}{ Balady } & $10 \mathrm{~cm}$ & $15.75^{\mathrm{a}}$ & $23.59^{\mathrm{a}}$ & $15.41^{\mathrm{a}}$ & $66.91^{\mathrm{b}}$ \\
\hline & $15 \mathrm{~cm}$ & $14.00^{\mathrm{a}}$ & $25.08^{\mathrm{b}}$ & $16.25^{\mathrm{a}}$ & $68.98^{\mathrm{a}}$ \\
\hline \multirow[t]{2}{*}{ Sids-40 } & $10 \mathrm{~cm}$ & $16.42^{\mathrm{a}}$ & $24.39^{c}$ & $15.41^{\mathrm{a}}$ & $67.25^{\mathrm{b}}$ \\
\hline & $15 \mathrm{~cm}$ & $13.92^{\mathrm{a}}$ & $24.20^{\mathrm{d}}$ & $16.01^{\mathrm{a}}$ & $67.32^{\mathrm{b}}$ \\
\hline
\end{tabular}

Means followed by the same letters were not statistical significantly differed according to Duncan's multiple range test. 
Table 15. Interaction effect between cultivars and cropping system on sugar beet growth traits

\begin{tabular}{|c|c|c|c|c|c|}
\hline \multicolumn{2}{|c|}{ Cultivars X Cropping system } & $\begin{array}{c}\text { Leaves number } \\
\text { plant }^{-1}\end{array}$ & \multirow{2}{*}{$\begin{array}{c}\text { Leaves weight } \\
\text { plant }^{-1}\end{array}$} & \multirow[t]{2}{*}{$\begin{array}{c}\text { Root weight } \\
\text { plant }^{-1}\end{array}$} & \multirow[t]{2}{*}{$\begin{array}{c}\text { Root diameter } \\
\text { plant }^{-1}\end{array}$} \\
\hline \multicolumn{3}{|c|}{$2014 / 2015$} & & & \\
\hline \multirow{4}{*}{ Balady } & Sole & $33.00 \mathrm{a}$ & $383.3 \mathrm{a}$ & $833.3 \mathrm{a}$ & $26.73 a$ \\
\hline & One row & $31.67^{\mathrm{a}}$ & $345.8^{\mathrm{a}}$ & $766.7^{\mathrm{a}}$ & $24.4^{\mathrm{b}}$ \\
\hline & Two rows & $28.67^{\mathrm{a}}$ & $262.5^{\mathrm{a}}$ & $650.0^{\mathrm{a}}$ & $22.4^{\mathrm{c}}$ \\
\hline & Three rows & $27.00^{\mathrm{a}}$ & $175.0^{\mathrm{a}}$ & $466.7^{\mathrm{a}}$ & $18.23^{\mathrm{e}}$ \\
\hline \multirow[t]{3}{*}{ Sids- 40} & One row & $31.83^{\mathrm{a}}$ & $341.0^{\mathrm{a}}$ & $741.7^{\mathrm{a}}$ & $22.07^{\mathrm{c}}$ \\
\hline & Two rows & $29.00^{\mathrm{a}}$ & $250.0^{\mathrm{a}}$ & $641.7^{\mathrm{a}}$ & $20.57^{\mathrm{d}}$ \\
\hline & Three rows & $27.17^{\mathrm{a}}$ & $208.3^{\mathrm{a}}$ & $466.6^{\mathrm{a}}$ & $16.73^{\mathrm{f}}$ \\
\hline \multicolumn{6}{|c|}{$2015 / 2016$} \\
\hline \multirow{4}{*}{ Balady } & Sole & $30.33 a$ & $358.3 \mathrm{a}$ & 800 & $25.33 \mathrm{a}$ \\
\hline & One row & $29.33^{\mathrm{b}}$ & $339.7^{\mathrm{a}}$ & $719.2^{\mathrm{a}}$ & $23.00^{\mathrm{b}}$ \\
\hline & Two rows & $26.83^{\mathrm{d}}$ & $247.0^{\mathrm{c}}$ & $625.0^{\mathrm{a}}$ & $21.00^{\mathrm{c}}$ \\
\hline & Three rows & $25.50 \mathrm{e}$ & $156.7^{\mathrm{b}}$ & $439.2^{\mathrm{a}}$ & $17.33^{\mathrm{e}}$ \\
\hline \multirow[t]{3}{*}{ Sids- 40} & One row & $28.33^{\mathrm{c}}$ & $309.0^{\mathrm{b}}$ & $681.7^{\mathrm{a}}$ & $20.67^{\mathrm{c}}$ \\
\hline & Two rows & $27.00^{\mathrm{d}}$ & $233.8^{\mathrm{ab}}$ & $616.7^{\mathrm{a}}$ & $19.17^{\mathrm{d}}$ \\
\hline & Three rows & $24.67^{\mathrm{f}}$ & $199.2^{\mathrm{b}}$ & $432.8^{\mathrm{a}}$ & $15.33^{\mathrm{f}}$ \\
\hline
\end{tabular}

Means followed by the same letters were not statistical significantly differed according to Duncan's multiple range test.

Table 16. Interaction effect between cultivars and cropping system on sugar beet yield quality traits

\begin{tabular}{|c|c|c|c|c|c|}
\hline \multicolumn{2}{|c|}{ Cultivars X Cropping system } & $\begin{array}{c}\text { root length } \\
(\mathrm{cm})\end{array}$ & \multirow{2}{*}{$\begin{array}{l}\begin{array}{c}\text { yield weight } \\
\text { (ton) }\end{array} \\
15\end{array}$} & \multirow[t]{2}{*}{ Sucrose\% } & \multirow[t]{2}{*}{ Purity \% } \\
\hline \multicolumn{3}{|c|}{$2014 / 2015$} & & & \\
\hline \multirow{4}{*}{ Balady } & Sole & 14.98 & 29.00 & 16.80 & $73.71 \mathrm{a}$ \\
\hline & One row & $14.98^{\mathrm{a}}$ & $25.14^{\mathrm{a}}$ & $16.65^{\mathrm{a}}$ & $69.18^{\mathrm{a}}$ \\
\hline & Two rows & $15.81^{\mathrm{a}}$ & $23.81^{\mathrm{a}}$ & $15.78^{\mathrm{a}}$ & $67.3^{\mathrm{a}}$ \\
\hline & Three rows & $17.64^{\mathrm{a}}$ & $21.76^{\mathrm{a}}$ & $15.17^{\mathrm{a}}$ & $65.5 \mathrm{a}$ \\
\hline \multirow[t]{3}{*}{ Sids-40 } & One row & $14.98^{\mathrm{a}}$ & $25.01^{\mathrm{a}}$ & $16.40^{\mathrm{a}}$ & $68.59^{\mathrm{a}}$ \\
\hline & Two rows & $16.31^{\mathrm{a}}$ & $23.96^{\mathrm{a}}$ & $15.64^{\mathrm{a}}$ & $66.27^{\mathrm{a}}$ \\
\hline & Three rows & $17.2^{\mathrm{a}}$ & $22.29^{\mathrm{a}}$ & $14.92^{\mathrm{a}}$ & $64.52^{\mathrm{a}}$ \\
\hline \multicolumn{6}{|c|}{$2015 / 2016$} \\
\hline \multirow{4}{*}{ Balady } & Sole & $14.00 \mathrm{a}$ & $28.17 \mathrm{a}$ & $16.62 \mathrm{a}$ & $72.73 a$ \\
\hline & One row & $14.00^{\mathrm{a}}$ & $24.41^{\mathrm{a}}$ & $16.13^{\mathrm{a}}$ & $68.19^{\mathrm{a}}$ \\
\hline & Two rows & $14.83^{\mathrm{a}}$ & $23.40^{\mathrm{a}}$ & $15.55^{\mathrm{a}}$ & $66.31^{\mathrm{a}}$ \\
\hline & Three rows & $16.67^{\mathrm{a}}$ & $21.37^{\mathrm{a}}$ & $15.00^{\mathrm{a}}$ & $64.56^{\mathrm{a}}$ \\
\hline \multirow[t]{3}{*}{ Sids-40 } & One row & $15.03^{\mathrm{a}}$ & $24.32^{\mathrm{a}}$ & $16.11^{\mathrm{a}}$ & $67.60^{\mathrm{a}}$ \\
\hline & Two rows & $15.41^{\mathrm{a}}$ & $23.12^{\mathrm{a}}$ & $15.44^{\mathrm{a}}$ & $65.28^{\mathrm{a}}$ \\
\hline & Three rows & $16.23^{\mathrm{a}}$ & $21.58^{\mathrm{a}}$ & $14.67^{\mathrm{a}}$ & $63.53^{\mathrm{a}}$ \\
\hline
\end{tabular}

Means followed by the same letters were not statistical significantly differed according to Duncan's multiple range test. 
Table 17.Interaction effect between intra-row spacing and cropping system on sugar beet growth traits

\begin{tabular}{|c|c|c|c|c|c|}
\hline \multicolumn{2}{|c|}{ Distances X Cropping system } & \multirow{2}{*}{$\begin{array}{c}\text { Leaves } \\
\text { number plant } \\
-1 \\
2014 / 2015\end{array}$} & \multirow[t]{2}{*}{$\begin{array}{c}\text { Leaves } \\
\text { weight plant }^{-1}\end{array}$} & \multirow[t]{2}{*}{$\begin{array}{l}\text { Root weight } \\
\text { plant }^{-1}\end{array}$} & \multirow[t]{2}{*}{$\begin{array}{c}\text { Root } \\
\text { diameter }^{-1} \text { plant }^{-1} \\
\end{array}$} \\
\hline & & & & & \\
\hline & Sole & $33.0 \mathrm{a}$ & $383.33 \mathrm{a}$ & $833.3 \mathrm{a}$ & $26.0 \mathrm{a}$ \\
\hline \multirow[t]{3}{*}{$10 \mathrm{~cm}$} & One row & $30.50^{\mathrm{b}}$ & $316.67^{\mathrm{b}}$ & $708.33^{\mathrm{b}}$ & $23.17^{\mathrm{b}}$ \\
\hline & Two rows & $27.83^{\mathrm{c}}$ & $233.33^{\mathrm{c}}$ & $625.00^{\mathrm{c}}$ & $21.50^{\mathrm{c}}$ \\
\hline & Three rows & $25.83^{\mathrm{d}}$ & $141.67^{\mathrm{e}}$ & $425.00^{\mathrm{e}}$ & $16.00^{\mathrm{d}}$ \\
\hline \multirow[t]{5}{*}{$15 \mathrm{~cm}$} & One row & $33.00^{\mathrm{a}}$ & $370.8^{\mathrm{a}}$ & $800.00^{\mathrm{a}}$ & $24.33^{\mathrm{ab}}$ \\
\hline & Two rows & $29.83^{\mathrm{b}}$ & $279.17^{\mathrm{b}}$ & $666.67^{\mathrm{b}}$ & $22.00^{\mathrm{abc}}$ \\
\hline & Three rows & $28.33^{\mathrm{c}}$ & $241.67^{\mathrm{c}}$ & $508.33^{\mathrm{c}}$ & $18.50^{\mathrm{d}}$ \\
\hline & & $2015 / 2016$ & & & \\
\hline & Sole & $30.33 \mathrm{a}$ & $358.33 \mathrm{a}$ & $800 \mathrm{a}$ & $28.33 \mathrm{a}$ \\
\hline \multirow[t]{3}{*}{$10 \mathrm{~cm}$} & One row & $27.50^{\mathrm{b}}$ & $303.17^{\mathrm{b}}$ & $644.17^{\mathrm{b}}$ & $21.50^{\mathrm{b}}$ \\
\hline & Two rows & $26.50^{\mathrm{c}}$ & $218.83^{\mathrm{c}}$ & $600.83^{c}$ & $20.33^{c}$ \\
\hline & Three rows & $23.83^{\mathrm{d}}$ & $130.83^{\mathrm{e}}$ & $406.50^{\mathrm{e}}$ & $15.17 d$ \\
\hline \multirow[t]{3}{*}{$15 \mathrm{~cm}$} & One row & $30.17^{\mathrm{a}}$ & $345.00^{\mathrm{a}}$ & $756.67^{\mathrm{a}}$ & $22.17^{\mathrm{b}}$ \\
\hline & Two rows & $27.33^{\mathrm{b}}$ & $262.00^{\mathrm{b}}$ & $640.83^{b c}$ & $19.83^{b}$ \\
\hline & Three rows & $26.33^{c}$ & $225.00^{\mathrm{c}}$ & $465.5^{\mathrm{d}}$ & $17.50^{\mathrm{c}}$ \\
\hline
\end{tabular}

Means followed by the same letters were not statistical significantly differed according to Duncan's multiple range test.

Table 18. Interaction effect between intra-row spacing and cropping system on sugar beet yield quality traits

\begin{tabular}{|c|c|c|c|c|c|}
\hline \multicolumn{2}{|c|}{ Distones $\mathbf{Y}$ Cranning cratom } & $\begin{array}{c}\text { root } \\
\text { length }(\mathrm{cm})\end{array}$ & $\begin{array}{c}\text { yield } \\
\text { weight(ton) }\end{array}$ & \multirow[t]{2}{*}{ Sucrose\% } & \multirow[t]{2}{*}{ Purity $\%$} \\
\hline \multicolumn{4}{|c|}{$2014 / 2015$} & & \\
\hline \multirow{4}{*}{$10 \mathrm{~cm}$} & Sole & 14.98 & 29 & 16.80 & 73.71 \\
\hline & One row & $15.83^{\mathrm{a}}$ & $24.50^{\mathrm{a}}$ & $16.00^{\mathrm{a}}$ & $68.14^{\mathrm{b}}$ \\
\hline & Two rows & $17.33^{\mathrm{b}}$ & $23.47^{\mathrm{b}}$ & $15.24^{\mathrm{b}}$ & $65.84^{\mathrm{b}}$ \\
\hline & Three rows & $19.17^{\mathrm{b}}$ & $21.25^{\mathrm{b}}$ & $14.73^{\mathrm{b}}$ & $64.58^{\mathrm{b}}$ \\
\hline \multirow{3}{*}{$15 \mathrm{~cm}$} & One row & $12.67^{\mathrm{a}}$ & $25.65^{\mathrm{a}}$ & $17.04^{\mathrm{a}}$ & $69.62^{\mathrm{b}}$ \\
\hline & Two rows & $14.00^{\mathrm{b}}$ & $24.29^{\mathrm{b}}$ & $16.17^{b}$ & $67.73^{\mathrm{b}}$ \\
\hline & Three rows & $17.00^{\mathrm{b}}$ & $22.80^{\mathrm{b}}$ & $15.35^{\mathrm{b}}$ & $65.48^{\mathrm{b}}$ \\
\hline \multicolumn{6}{|c|}{ 2015/2016 } \\
\hline \multirow{3}{*}{$10 \mathrm{~cm}$} & Sole & 14 & 28.17 & 16.62 & 72.73 \\
\hline & One row & $15.83 \mathrm{a}$ & $23.91^{b}$ & $15.47^{\mathrm{a}}$ & $67.15^{\mathrm{b}}$ \\
\hline & Two rows & $16.67^{\mathrm{b}}$ & $23.01^{\mathrm{b}}$ & $15.00^{\mathrm{b}}$ & $64.86^{\mathrm{b}}$ \\
\hline \multirow{4}{*}{$15 \mathrm{~cm}$} & Three rows & $17.83^{\mathrm{b}}$ & $20.88^{\mathrm{c}}$ & $14.54^{\mathrm{b}}$ & $63.59^{b}$ \\
\hline & One row & $13.20^{\mathrm{a}}$ & $24.82^{\mathrm{b}}$ & $16.77^{\mathrm{a}}$ & $68.64^{\mathrm{b}}$ \\
\hline & Two rows & $13.58^{\mathrm{b}}$ & $23.51^{\mathrm{b}}$ & $16.00^{\mathrm{b}}$ & $66.74^{\mathrm{b}}$ \\
\hline & Three rows & $15.07^{\mathrm{b}}$ & $22.07^{\mathrm{c}}$ & $15.13^{\mathrm{b}}$ & $64.49^{\mathrm{b}}$ \\
\hline
\end{tabular}

Means followed by the same letters were not statistical significantly differed according to Duncan's multiple range test.

On the other hand the higher value of root length parameter was obtained from sugar beet intercropped with garlic at $10 \mathrm{~cm}$ intra- row spacing three garlic rows (19.17 and 17.83) in two seasons respectively compared with sole sugar beet and intercropped treatment irrespective of garlic cultivars tested.
4- Effect the interaction between garlic cultivars, intra-row spacing and cropping system:

The results in Tables $19 \& 20$ showed that, effect of the interaction between garlic cultivars, intra-row spacing and cropping systems on growth, yield and quality of sugar beet in 2014/2015and 2015/2016. There were no significant effects on all growth, yield and its components of Sugar beet in two seasons except, root length and purity $\%$ in two seasons and yield weight in 
$2^{\text {nd }}$ season. Sole sugar beet accounted significantly higher in yield weight (29 and 28.17) and purity (73.71 and $72.73 \%$ ) respectively in two seasons compared to other treatments followed by sugar beet intercropped with Balady cv. at $15 \mathrm{~cm}$ intra-row spacing in one row for yield weight(25.93and 25.47) and purity (70.05 and $69.54 \%$ ), respectively in two seasons. Meanwhile sugar beet intercropped with Sids- $40 \mathrm{cv}$. at $10 \mathrm{~cm}$ intra-row spacing in three rows significantly lower in yield weight (21.51 and 21.40). While, sugar beet intercropped with Sids-40cv. at $15 \mathrm{~cm}$ intra-row spacing three rows significantly lower in purity (63.39 and 63.21\%) respectively in two seasons. Root length of sugar beet intercropped with Sids- $40 \mathrm{cv}$. at $10 \mathrm{~cm}$ in three rows recorded higher value (19.33 and 18.67) compared to other treatments, meanwhile, lower value (13.31 and 12.33) was obtained from sugar beet intercropped with Balady cv. at $15 \mathrm{~cm}$ intra-row spacing one row respectively in two seasons the rest of treatments were intermediate.

\section{*Land equivalent ratio (LER):}

Data in table 21 showed the treatments effect on land use efficiency of garlic intercropped with sugar beet. The data revealed that the highest value of LER was obtained from intercropping garlic Cultivar Balady at $10 \mathrm{~cm}$ intra-plant spacing with three rows on the top of sugar beet bed flowed with the same cultivars at $15 \mathrm{~cm}$ intra-row spacing with two rows intercropped as mean of two seasons. On the other hand, the lowest value of LER was obtained when Sids- 40 at $15 \mathrm{~cm}$ and planted at one row on the top of sugar beet bed as mean of two seasons. These results were in the same line with those obtained by Abou Khadra et al. (2013), and El-Shereif (2013) who stated that intercropping system recorded higher LER over sole cropping.

Table 19. Interaction effect between garlic cultivars, intra-row spacing and cropping system on sugar beet growth traits

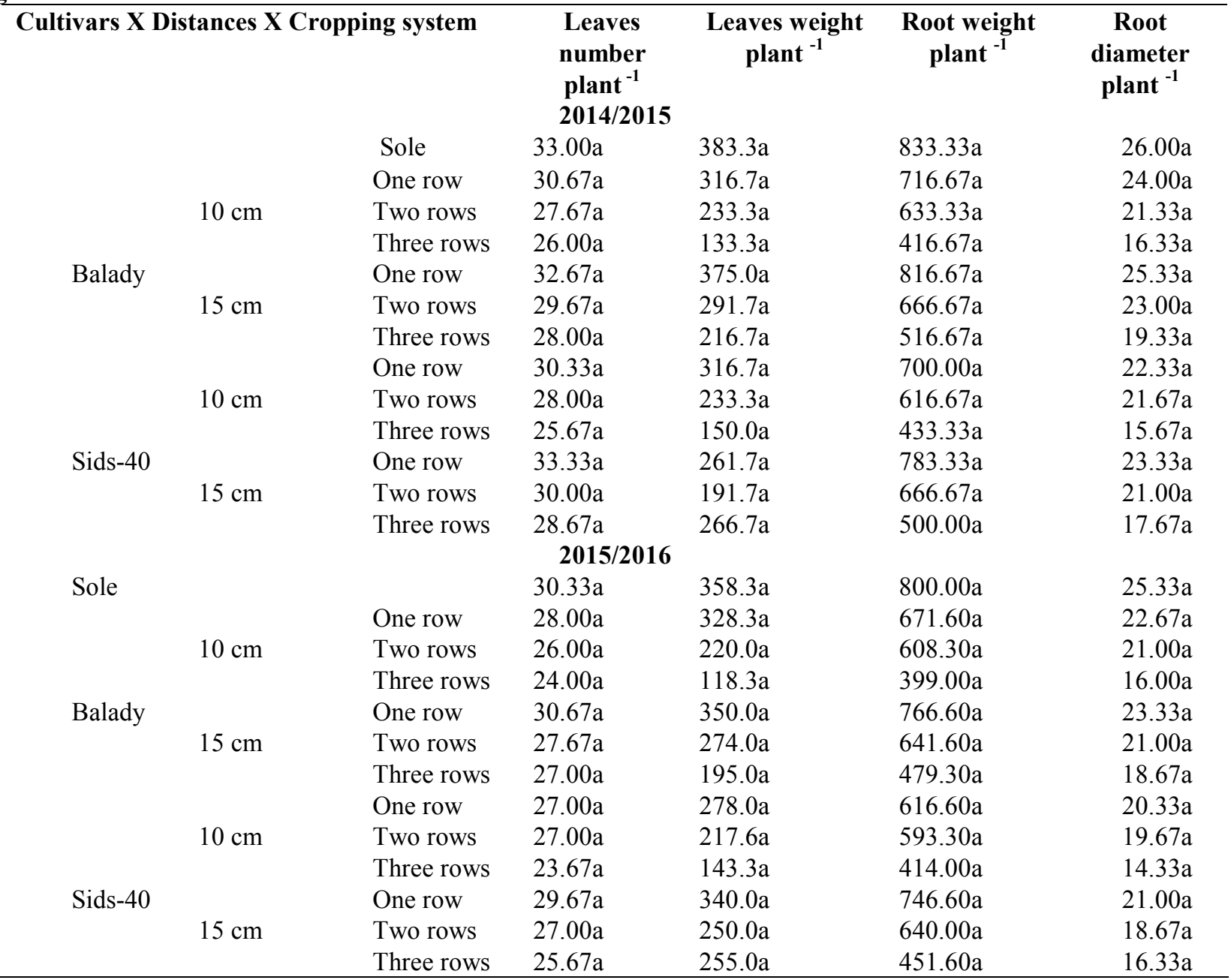

Means followed by the same letters were not statistical significantly differed according to Duncan's multiple range test. 
Table 20. Interaction effect between garlic cultivars, intra-row spacing and row cropping system on sugar beet yield and quality traits

\begin{tabular}{|c|c|c|c|c|c|c|}
\hline \multicolumn{3}{|c|}{ Cultivars x Distances x Cropping system } & \multirow{2}{*}{$\begin{array}{c}\begin{array}{c}\text { Root length } \\
(\mathrm{cm})\end{array} \\
2014 / 2015\end{array}$} & \multirow{2}{*}{$\begin{array}{c}\text { Yield weight } \\
\text { fad }^{-1} \text { (ton) }\end{array}$} & \multirow[t]{2}{*}{ Sucrose\% } & \multirow[t]{2}{*}{ Purity\% } \\
\hline & & & & & & \\
\hline & & Sole & $14.98 \mathrm{a}$ & $29.00 \mathrm{a}$ & $16.80 \mathrm{a}$ & $73.71 \mathrm{a}$ \\
\hline & $10 \mathrm{~cm}$ & One row & $16.65 \mathrm{a}$ & $24.35 \mathrm{~d}$ & $16.04 \mathrm{a}$ & $67.82 \mathrm{~cd}$ \\
\hline & & Two rows & $17.3 \mathrm{a}$ & $23.17 \mathrm{efg}$ & $15.34 \mathrm{a}$ & $65.74 \mathrm{ef}$ \\
\hline & & Three rows & $17.98 \mathrm{a}$ & $20.99 \mathrm{~h}$ & $14.90 \mathrm{a}$ & $64.32 \mathrm{~g}$ \\
\hline \multirow[t]{6}{*}{ Balady } & $15 \mathrm{~cm}$ & One row & $13.31 \mathrm{a}$ & $25.93 b$ & $17.26 \mathrm{a}$ & $70.53 b$ \\
\hline & & Two rows & $14.31 \mathrm{a}$ & $24.44 \mathrm{~cd}$ & $16.21 \mathrm{a}$ & $68.85 \mathrm{c}$ \\
\hline & & Three rows & $17.33 \mathrm{a}$ & $22.53 \mathrm{~g}$ & $15.43 \mathrm{a}$ & $66.77 \mathrm{de}$ \\
\hline & $10 \mathrm{~cm}$ & One row & $16.98 \mathrm{a}$ & $24.65 \mathrm{~cd}$ & $15.96 \mathrm{a}$ & $68.46 \mathrm{c}$ \\
\hline & & Two rows & $17.98 \mathrm{a}$ & $23.78 \mathrm{def}$ & $15.14 \mathrm{a}$ & $65.95 \mathrm{e}$ \\
\hline & & Three rows & $19.64 \mathrm{a}$ & $21.51 \mathrm{~h}$ & $14.56 \mathrm{a}$ & $64.83 \mathrm{fg}$ \\
\hline \multirow[t]{8}{*}{ Sids-40 } & $15 \mathrm{~cm}$ & One row & $15.04 \mathrm{a}$ & $25.36 \mathrm{bc}$ & $16.83 \mathrm{a}$ & $68.72 \mathrm{c}$ \\
\hline & & Two rows & $14.79 \mathrm{a}$ & $24.14 \mathrm{de}$ & $16.13 \mathrm{a}$ & $66.6 \mathrm{e}$ \\
\hline & & Three rows & $14.78 \mathrm{a}$ & $23.07 \mathrm{fg}$ & $15.27 \mathrm{a}$ & $64.2 \mathrm{~g}$ \\
\hline & & & $2015 / 2016$ & & & \\
\hline & & Sole & $14.00 \mathrm{a}$ & $28.17 \mathrm{a}$ & $16.62 \mathrm{a}$ & $72.73 a$ \\
\hline & & One row & $15.67 \mathrm{a}$ & $23.35 \mathrm{~d}$ & $15.22 \mathrm{a}$ & $66.83 \mathrm{~cd}$ \\
\hline & $10 \mathrm{~cm}$ & Two rows & $16.33 \mathrm{a}$ & $22.50 \mathrm{efg}$ & $15.01 \mathrm{a}$ & $64.75 \mathrm{ef}$ \\
\hline & & Three rows & $17.00 \mathrm{a}$ & $20.36 \mathrm{~h}$ & $14.76 \mathrm{a}$ & $63.34 \mathrm{~g}$ \\
\hline \multirow[t]{6}{*}{ Balady } & & One row & $12.33 \mathrm{a}$ & $25.47 b$ & $17.03 \mathrm{a}$ & $69.54 b$ \\
\hline & $15 \mathrm{~cm}$ & Two rows & $13.33 \mathrm{a}$ & $24.30 \mathrm{~cd}$ & $16.09 \mathrm{a}$ & $67.86 \mathrm{c}$ \\
\hline & & Three rows & $16.33 \mathrm{a}$ & $22.37 f$ & $15.24 \mathrm{a}$ & $65.78 \mathrm{de}$ \\
\hline & & One row & $16.00 \mathrm{a}$ & $24.47 \mathrm{~cd}$ & $15.72 \mathrm{a}$ & $67.47 \mathrm{c}$ \\
\hline & $10 \mathrm{~cm}$ & Two rows & $17.00 \mathrm{a}$ & $23.52 \mathrm{def}$ & $14.98 \mathrm{a}$ & $64.96 \mathrm{e}$ \\
\hline & & Three rows & $18.67 \mathrm{a}$ & $21.40 \mathrm{~h}$ & $14.31 \mathrm{a}$ & $63.84 \mathrm{fg}$ \\
\hline \multirow[t]{3}{*}{ Sids-40 } & & One row & $14.06 \mathrm{a}$ & $24.17 b c$ & $16.50 \mathrm{a}$ & $67.73 c$ \\
\hline & $15 \mathrm{~cm}$ & Two rows & $13.82 \mathrm{a}$ & $22.72 \mathrm{de}$ & $15.91 \mathrm{a}$ & $65.61 \mathrm{e}$ \\
\hline & & Three rows & $13.80 \mathrm{a}$ & $21.76 \mathrm{fg}$ & $15.02 \mathrm{a}$ & $63.21 \mathrm{~g}$ \\
\hline
\end{tabular}

Means followed by the same letters were not statistical significantly differed according to Duncan's multiple range test.

\section{* Economic evaluation:-}

The economic study of various treatment combinations are given in Table 21. The highest total gross return from Sids- $40 \mathrm{cv}$. at $15 \mathrm{~cm}$ intra-row spacing with two rows of garlic intercropped with sugar beet (27992 L.E/ fed.) closely followed by Balady cv (25995 L.E./ fed.) at $15 \mathrm{~cm}$ intra-row spacing and two rows on the top of sugar beet bed as mean of two seasons with a net income (20052 and 19395 L.E. / fed) respectively.

These results agree with Castellanos et al., (2004). Who said that, although plant yield increased when the higher plant density were used but the yield improvement increased plant stand is offset by the reduction in bulb size which severely affects quality and market value.

\section{CONCLUSION}

Eventually, Garlic cultivar (Balady or Sids-40) when planted at the intra-row spacing $15 \mathrm{~cm}$ and intercropping two garlic rows on the top of sugar beet bed could be the appropriate suggestion for increasing net income of farmers in North Middle Nile Delta region, Egypt. 
Table 21. Effects of garlic cultivars and intra-row spacing intercropped with sugar beet on LER and economic values

\begin{tabular}{|c|c|c|c|c|c|c|c|c|c|c|}
\hline \multicolumn{2}{|c|}{ Treatments } & \multirow[b]{2}{*}{$\begin{array}{l}\text { Cropping } \\
\text { system }\end{array}$} & \multicolumn{2}{|c|}{$\begin{array}{c}\text { Relative yield } \\
\text { (RY) }\end{array}$} & \multirow{2}{*}{ LER } & \multirow{2}{*}{$\begin{array}{l}\text { Gross } \\
\text { income } \\
\text { for } \\
\text { garlic }\end{array}$} & \multirow{2}{*}{$\begin{array}{l}\text { Gross } \\
\text { income } \\
\text { for } \\
\text { sugar } \\
\text { beet } \\
\end{array}$} & \multirow{2}{*}{$\begin{array}{l}\text { Total } \\
\text { income } \\
\text { L.E/fe } \\
\text { d.) }\end{array}$} & \multirow{2}{*}{$\begin{array}{l}\text { Total } \\
\text { Cost } \\
\text { L.E./ } \\
\text { fed. }\end{array}$} & \multirow{2}{*}{$\begin{array}{l}\text { Net } \\
\text { income }\end{array}$} \\
\hline Cultivars & Distances & & garlic & $\begin{array}{l}\text { sugar } \\
\text { beet }\end{array}$ & & & & & & \\
\hline \multirow{5}{*}{ Balady } & \multirow{3}{*}{$10 \mathrm{~cm}$} & One row & 0.398 & 0.900 & 1.298 & 14340 & 9506 & 23846 & 6830 & 17016 \\
\hline & & Two rows & 0.770 & 0.722 & 1.492 & 20790 & 7623 & 26104 & 7680 & 18424 \\
\hline & & Three rows & 1.043 & 0.509 & 1.553 & 18780 & 5381 & 24161 & 8520 & 15641 \\
\hline & \multirow{3}{*}{$15 \mathrm{~cm}$} & One row & 0.338 & 0.975 & 1.313 & 12180 & 10300 & 22481 & 6640 & 15841 \\
\hline & & Two rows & 0.610 & 0.902 & 1.512 & 18300 & 9525 & 25995 & 6640 & 19355 \\
\hline \multirow{7}{*}{ Sids-40 } & & Three rows & 0.742 & 0.599 & 1.341 & 13350 & 6326 & 19676 & 7280 & 12396 \\
\hline & \multirow{3}{*}{$10 \mathrm{~cm}$} & One row & 0.377 & 0.940 & 1.317 & 15840 & 9926 & 25766 & 7250 & 18516 \\
\hline & & Two rows & 0.636 & 0.870 & 1.506 & 20025 & 9195 & 26995 & 8500 & 18495 \\
\hline & & Three rows & 0.896 & 0.547 & 1.442 & 18810 & 5775 & 24585 & 9750 & 14835 \\
\hline & \multirow{3}{*}{$15 \mathrm{~cm}$} & One row & 0.331 & 0.929 & 1.260 & 13920 & 9813 & 23734 & 6940 & 16794 \\
\hline & & Two rows & 0.591 & 0.886 & 1.478 & 20700 & 9362 & 27992 & 7940 & 20052 \\
\hline & & Three rows & 0.800 & 0.577 & 1.377 & 16800 & 6097 & 22898 & 8900 & 13998 \\
\hline
\end{tabular}

Total income for solid crops: Sugar beet: LE 10875 Garlic LE 30000

\section{REFERENCES}

Abdel Motagally, F. M. F. and A .K .Metwally 2014. Maximizing productivity by intercropping onion on sugar beet. Asian J. of Crop Sci., 6: 226-235.

Abdel Razzak,H.S. and El-Sharkawy .2013.Effect biofertilizer and humic acid applications on growth, yieled quality and storability of two garlic cultivars. Asian Journal of Crop Science 5 (1):48-64

Abd El-Zaher, Sh. R., M.S.H. Osman and A.Sh. Sahar. 2009. Effect of intercropping barley with sugar beet under different nitrogen fertilization levels on yield and yield components. Egypt. J. of Appl. Sci., 24 (6 B): 531 - 550.

Abou El-Magd, M.M., ,T.El-Sourbagy, S.M.Shehata . 2012. A comparative study on the productivity of four Egyptian garlic cultivars grown under various organic material in comparison to conventional chemical fertilizer. Australian Journal of Basic and Applied Sciences, 6(3):415-421.

Abou Khadra, S.H., A.E.B. Shaimaa, E.A.T. Salah and E.E.E. Dina. 2013. Effect of intercropping wheat with sugar beet on their productivity and land use. J.Agric. R.es. Kafr ElSheikh Univ., 39:37-53.

Ahmed, S.E., A.M. Abou-Salama, G.R. El-Naggar and F.M.F. Abdel-Motagally (2009). Studies on legume-black cumin intercropping. Egypt. J. Appl. Sci., 24: 553-563.

Alam M.S., M.A.Rahim, M.A.H.Bhuyan, P.W. Simon, and M.A.Malek .2010. Effect of spacing on growth and yield of two lines of garlic under dry land condition. J. Agrofor. Environ. 4 (2): 151-154.

Al-Otayk, S., M. Z.El-Shinawy, and M. I. Motawei. 2008. Vareation in Productive Characteristics and Diversity Assessment of Garlic Cultivars and Lines Using DNA Markers. JKAU: Met., Env. \& Arid Land Agric. Sci., Vol. 20 No. 1, pp: 63-79.
Aly, Shereen H. 2010. Growth and yield of some garlic (Allium sativum L) ecotype as affected by different cultural practices under Assiut conditions. Ph.D. Thesis, Fac. Agric. Assiut Univ., pp: 137.

Amin, M., M.S. Bhuiyan, A.H.M.A. Faisal, I.S.M. Farhad and M.A. Rahaman . 2011. Crop Adaptation in Saline Soils of Noakhali (I. Crop performance). Bangladesh Agron. J., 14(1 and 2): 43-52.

Ammar, A.Y.M. 2007. Some studies on improving garlic producyivity. M. Sc. Thesis, Fac. Agric., El-Minia Univ., Egypt

Anwar, E. A. I., and Gouda. 2012. Evaluation of Some Garlic (Allium sativum L.) Cultivars Grown Under Mansoura Region Conditions. Res. J. of Agric. and Bio. Sci., 8(5): 407-410.

Azza E. Kh., and H. H.Naglaa 2016. Productivity of Garlic (Allium Sativum) Varieties Under Different Densities And Weed Control Treatments. boil. Sci.vol. 11(3): 335-358.

Baghalian, K., S.A. Ziai, M. R. Naghavi, A. H.Naghdi, A.Khalighi. 2005.. Evaluation of alliicin content and botanical traits I Iranian garlic (Allium sativum L) Ecotypes. Sci. Hort. 103: 155-166.

Castellanos, J.Z., P. Vargas-Tapia, J.L.Ojodeagua, G.Hoyos. 2004. Garlic productivity and profitability as affected by seed clove size, planting density and planting method. HortScience 39: 1272-1277.

Castillo, J.E., L. Lopez-Bellido, E.J.Fernandez, F.J.Lopez. 1996. Influence of planting geometry on growth, yield and quality of rain fed garlic (Allium sativum L.) cultivated under Mediterranean conditions. Journal of Horticultural Science 71: 867-879.

Dayi, R.U. 2008. Effects of NPK Fertilizer and Intra- row spacing on the growth and yield of garlic (Allium sativum 
L.). The Second National Conference of the Joint CST of Hassan Usman Katsina Polytechinc 27 - 30 th November.

Djordje Moravčević1*, Vukašin Bjelić1, Dubravka Savić1, Jelica Gvozdanović Varga2, Damir Beatović1, Slavica Jelačić1 and Vlade Zarić1. ( 2011). Effect of plant density on the characteristics of photosynthetic apparatus of garlic (Allium sativum var. vulgare L.)African Journal of Biotechnology Vol. 10(71), pp. 15861- 15868.

Donald, C. M. 1963. Competition among crop and pasture peanuts. Adv. Agron., 15: 1-118.

Duncan, D. B. 1955. Multiple range and multiple $\mathrm{F}$ test. Biometrics, 11:1-42.

Džamić, R., M. Nikolić, R.Stikić, Z.Jovanović. 2001. Fiziologija biljaka (praktikum, drugo izdanje). Naučna knjiga, Beograd, pp. 44-45. Steel, R. G. D. and Jour. H. Torrie. 1980. Principles and Procedures of Statistics. Mc. Graw Hill Book Company Inc. New York, 481 pp.

ELeshmwiy,K.H., L.M. ElSharif, H.B.Hassan and A.M. Saafan. 2010. Potentials of the economic expansion in the production and export of egyption garlic. Nat.Sci., 8:279287.

EL- Nagar, M.M., and S.S.M. EL-Zohiri. 2015. Physiological and Biotechnological studies on some Local and Foreign Garlic Genotypes.PARIPEX-INDIAN JOURANAL OF RESEARCH VOLUME 7:6-11.

El-Sarag, E.I. 2009. Maximizing sugar beet yield, quality and water use efficiency using some agriculture practices under North Sinai conditions. Bull. Fac. Agric., Cairo. Univ., 60(2): 155-167.

El-Shamy, Moshira. A, E.A.A. Moursi, and A. M. Mona, ELMansoury. 2015. Maximizing Water Productivity By Intercropping Onion on Surgar Beet In The North Middle Nile Delta Region. J. Soil Sci. and Agric . Eng., Mansoura Univ.,.6:961-982

El-Shereif , Dina , E.M. 2013. Effect of intercropping faba bean and wheat on sugar beet yield and its components. MSc, Thesis ,Kaferelsheikh Univ. Egypt.

FAO. 2012. Food and Agriculture Organization database for 2012 season

Gomez, K.A., and A.A. Gomez. 1984. Statistical procedures for agricultural research. 2nd, (ed). Jon willey and Sons, New York, U.S.A.

Hussein, M .M .M., and S. A. Metwally 2012. Effect of intercropping flax in different seeding rates in fields of sugar beet under nitrogen fertilization levels on yield, quality and land use efficiency attributes. Zagazig J. Agric. Res., 39: 9-29.

Hussein, N.S., H.M. El-Saeid, and E.A. Omer .1995. Development of growth and yield of some lines of Chinese garlic, Egypt. J. Hort., 22(1) 19-23.

Ibrahim, E.M., M.M.A.Badr and SH.R.Abd El-Zaher.2008. Response of some intercropping system of wheat with sugar beet to bio-mineral nitrogenous fertilization. Proceedings, The Second Field Crop Conference FCRI, ARC,Giza,Egypt,435-451.
Jabbar, A., I.H. Bhatti, A. Rehman and S.N. Wains. 2010. Effect of different rice-based intercropping systems on rice grain yield and residual soil fertility. Pakistan Journal of Botany 42:2339-2348.

Le- Decote, A. 1971. Commercial determination of sugar in the beet root wsing the sachs. Ledocte process. Int. Sug. J. 29, 488-492.

Lomte, M. H. and R. S.Dabhade. 1990. Intercropping studies in rainy season sorghum. Indian J. Agron., 3: 56-59.

Mahmoud, E.A., M.A. Hassanin, I.R. Emara Eman. 2012. Effect of organic and mineral nitrogenous fertilizers and plant density on yield and quality of sugar beet (Beta vulgaris L.). Egypt. J. Agron., 34(1): 89-103.

Masri, M.I., B.S.B. Ramadan, A.M.A. El-Shafai and M.S. ElKady. 2015. Effect of water stress and fertilization on yield and quality of sugar beet under drip and sprinkler irrigation systems in sandy soils. Int. J. Agric. Sci., 5(3): 414- 425.

Masri, M.I. and S.A. Safina. 2015. Agro-economic impact of intercropping canola and onion on some sugar beet varieties under different nitrogen rates. J. plant Production, Mansoura Univ., Vol. 6(10): 1661-1678

Mc Ginnus, R. A. 1982. Sugar Beet Technology, 3rd. Sugar beet Development Foundation, Fort Collins. PP.855, Colo, USA.

Menezes, S. M. J. A. D. E., R. E. D. E.Novais, H. L.Santos, and L.M. A.Dos. 1974. The effect of nitrogen fertilization, plant spacing and mulching on the yield of garlic cultivar Amarante. Tevista Res., 21 (115): 203- 212 [Cited from Hort. Abstr., 45 (9): 568, 1975]

Mohamed. S.I.A. 2004. Some studies to improve garlic productivity. Ph. D. Thesis, Fac. Agric., Minia Univ., ElMinia, Egypt.

Moravčević D., V.Bjelić1, D.Savić1, J.G.Varga, D.Beatović, S.Jelačić and V.Zarić. 2011. Effect of plant density on the characteristics of photosynthetic apparatus of garlic (Allium Sativum var. vulgare L.) African Journal of Biotechnology Vol. 10(71), pp. 15861-15868.

Moustafa, Y.M.M., S.S. Latif, Abd G.F. El-Naem, H.M.H. foully and S.I.Ahmed. 2009. Performance of new imported foreign garlic genotypes grown under the Egyptian conditions. Egypt. J. Agric. Res., 87(1): 219243.

Muro, J., I.Ignacio, C. Lamsfus, A.Fernandez Militino. 2000. Effect of defoliation on garlic yield. Scientia Horticulturae 86: 161-167.

Naruka I.S. and. R. S.Dhaka. 2001. Effect of row spacing and nitrogen fertilization on growth, yield and composition of bulb in garlic (Allium sativum L.) cultivars. Journal of Spices and Aromatic Crops 10 (2) : 111-117.

Nassar, S. H. , S.Moustapha, Sh. Foda, M. Gheta and S. Ghebrial . 1972. A better garlic variety export . Agric. Res. 50(4) 47-58. 
Olfati J.A., M.B.M.Najafabadi, and M.Rabiee. 2016. Between row spacing and local accession on the yield and quality of garlic. Comunicata Scientiae 7(1)

Om, H. and Srivastava, R. P. 1977. Performance of different locally selected garlic clove. Prog. Hort. 8(4): 69-76.

Osman, S.A., Y.M.M. Moustafa. 2009. Horticultural and cytogenetical characteristics of some Egyptian and foreign garlic cultivars. African Crop Science Conference Proceedings, 9: 459-465.

Panse, R. P.K., Jain A. Gupta and D. S. Sasode. 2013. Morphological vareability and character association in diverse collection of garlic germplasm. African Journal of Agricultural Research, 8(23): 2861-2869.

Purewal, S.S. and K.S.Daragan. 1961. Effect of fertilizers and sapacing on the development and yield of garlic (Allium sativum L.). Indian J.Agron. 5:262-268.

Rahim, M. A., M. A.Siddique, and M. M.Hossain. 1984. Effect of time of planting, mother bulb size and plant density on the yield of garlic. Bangladesh J. Agril. Res. 9 (2): 112-118.

Sandhu S.S, P.S. Brar, and R.K.Dhall. 2015. Vareability of agronomic and quality characteristics of garlic (Allium sativum L.) ecotypes. SABRA. Journal of Breeding and Genetics 47 (2) 133-142.

Sarkar, R. K., D.Shit, and A.Chakraborthy. 1995. Yield and economics of pigeonpea based intercropping system on rainfed upland of Chotanagpur plateau. Indian J. Agron., 40: 30-34.
Schoofs, A., and M.H. Entz. 2000. Influence of annual forages on weed dynamics in a cropping system. Can. J. Plant Sci., 80: 187-198.

Singh V., A. Kumar, and H.S.Sirohi. 1995. Effect of spacing t on the growth and yield of garlic. Indian J. Agric. Res. 29 : 153-156.

Sohier, M.M. Ouda. 2001. Response of sugar beet to N and K fertilizers levels under sandy soil conditions. Zagazig J. Agric. Res., 28(2): 275-297

Teasdale, J.R. 1998. Cover plants, smother plants, and weed management. p. 247-270. In: (Eds.): J.L. Hatfield et al., Integrated weed and soil management. Ann Arbor Press, Chelsea, MI

Toaima, S.E.A. 2006. Response of onion, Feba bean and wheat to intercropping with polder beet under different fertilizer levels of NPK. Minufiya. J. Agric. Res. Egypt, 31 (4): $939-956$.

Toaima, S.E.A., K.A. El-Douby and A.I. Nafei. 2000. Intercropping sugar beet with minor crops. Intensification Treatments, Sugar Crops, 21(3): 23-25.

Valadez LA. 1992. Onion (Allium cepa L.) and garlic (Allium sativum L.) production in Mexico. Onion Newsletter for the Tropics. No. 7.

Willy, R. W. And S.O. Osiur. 1972. Studies on mixtures of maize and beans phas+eolus vulgaris with particular reference to plant population. J. Agricu. Sci., 79:519. 


\section{الملخص العربي}

تاثير مسافات الزراعة ونظم التحميل مع بنجر السكر على النمو والمحصول والجودة في صنفين من اصناف الثوم

$$
\text { نجلاء حماده حسين، مشيره احمد الثامى }
$$

بينما اعطى الصنف سدس • ؛ اعلى القيم فى باقى الصفات

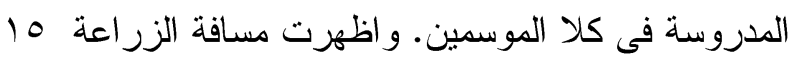

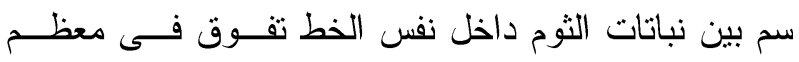

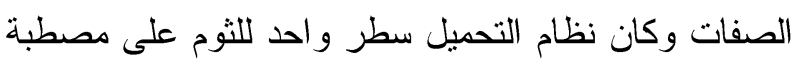

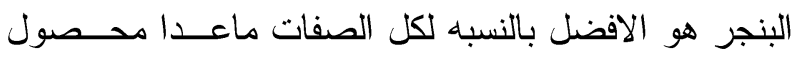

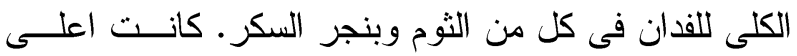

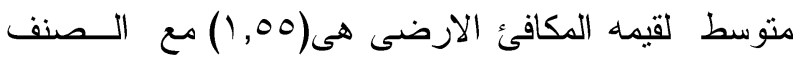

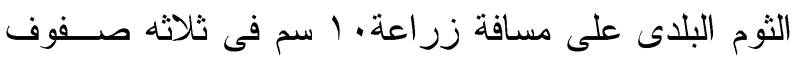

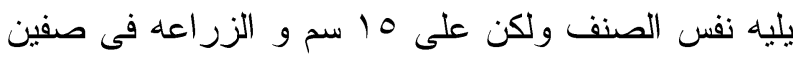
(1,01)

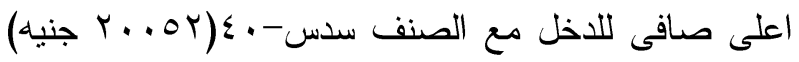

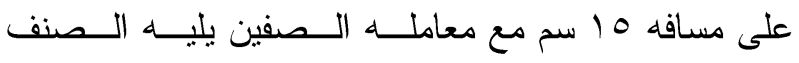

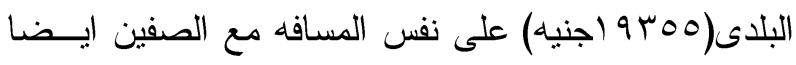
وذللك كمتوسط عام للموسمين.

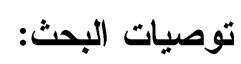

زر اعه اي من صنفين الثوم سدس-، ــ أو البلدى علـى

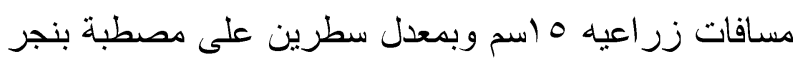

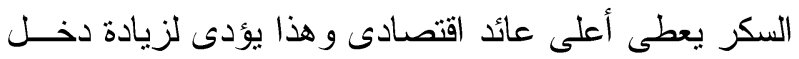
المز ارع في منطقة شمال الدلتا.
أجريت تجربه حقليه بمزرعه محطة بحوث البساتين بـسخا

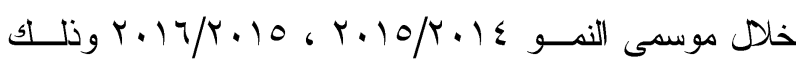

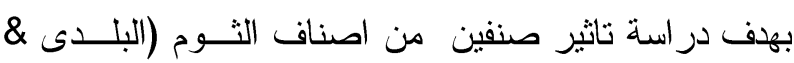

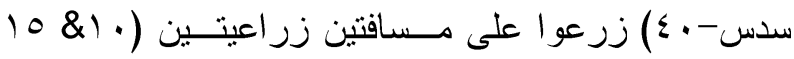
سم) تحت ثلاث نظم تحميل(تحميل ثوم مع بنحــر الـسكر بحيث يزرع سطر واحد فقط من الثوم \& تحميل ســطرين

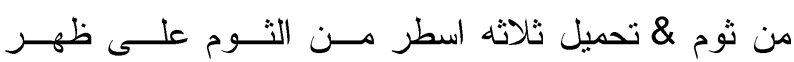
المصطبة مع بنحر السكر بحيث يكون بنجر السكر منزرع بالمعدل الموصى به) كذلك زر اعة بنجر السكر و الثوم منفرد

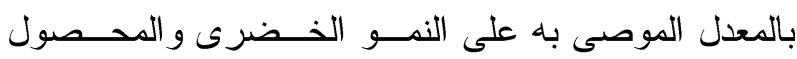
وجودته لكل من الثوم والبنجر كذلك تقييم المكافئ الارضى بـى

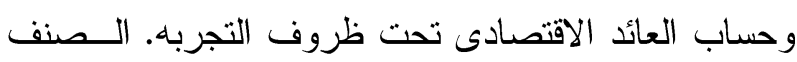
جلوريا كان الصنف المستخدم للمحصول الرئيسى (بنجـر

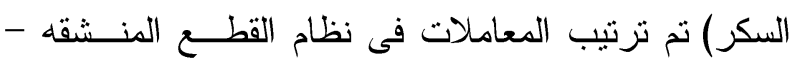

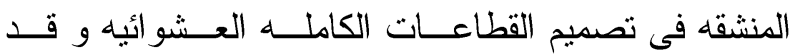
خصصت القطع الرئيسيه لاصناف الثوم و القطع المنـشقه لمسافات الزراعه و القطع تحت المنشقه الى نظم التحميل أهم النتائج يمكن تلخيصها كما يلى: اظهر الصنف البلدى للثوم تفوق فـى صــفات نـسبة الانبات، وارتفاع النبات، وقطر الراس وعدد الفـصوص. 